\title{
The Efficacy of an Energy-Restricted Anti-Inflammatory Diet for the Management of Obesity in Younger Adults
}

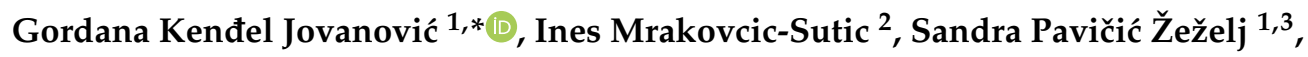 \\ Branislav Šuša ${ }^{4}$, Dario Rahelić ${ }^{5,6,7}$ and Sanja Klobučar Majanović ${ }^{8,9}$ \\ 1 Department of Health Ecology, Teaching Institute of Public Health of Primorsko-goranska County, \\ Krešimirova 52a, 51000 Rijeka, Croatia; sandrapz@medri.uniri.hr \\ 2 Department of Physiology, Immunology and Pathophysiology, Faculty of Medicine, University of Rijeka, \\ Braće Branchetta 20/1, 51000 Rijeka, Croatia; ines.mrakovcic.sutic@medri.uniri.hr \\ 3 Department of Health Ecology, Faculty of Medicine, University of Rijeka, Braće Branchetta 20/1, \\ 51000 Rijeka, Croatia \\ 4 General Hospital Pula, Santoriova 24a, 52100 Pula, Croatia; drbranislav.susa@gmail.com \\ 5 Vuk Vrhovac University Clinic for Diabetes, Endocrinology and Metabolic Diseases, \\ Merkur University Hospital, Dugi dol 4a, 10000 Zagreb, Croatia; dario.rahelic@gmail.com \\ 6 School of Medicine, University of Zagreb, Šalata ul. 2, 10000 Zagreb, Croatia \\ 7 School of Medicine, Josip Juraj Strossmayer University of Osijek, 31000 Osijek, Croatia \\ 8 Department of Endocrinology, Diabetes and Metabolic Diseases, Clinical Hospital Centre Rijeka, \\ Krešimirova 42, 51000 Rijeka, Croatia; sanja.klobucar@medri.uniri.hr \\ 9 Department of Internal Medicine, Faculty of Medicine, University of Rijeka, Braće Branchetta 20/1, \\ 51000 Rijeka, Croatia \\ * Correspondence: gordana.kendel-jovanovic@zzjzpgz.hr; Tel.: +358-51-358-751
}

Received: 28 October 2020; Accepted: 19 November 2020; Published: 22 November 2020

\begin{abstract}
There is growing evidence of the dietary impact on obesity-induced low-grade chronic inflammation and the associated chronic non-communicable diseases modification. We determined changes in body composition and cardiometabolic and inflammatory status of participants with obesity after 24 weeks of a dietary intervention based on an energy-reduced anti-inflammatory diet and examined the relationship of these changes with changes in the inflammatory potential of the diet. The anthropometric and body composition parameters of 81 participants (average age of 43 years, 74 women) were assessed. Metabolic status was determined using the glycemic and lipid statuses, and the cardiometabolic index and inflammatory status were determined using the concentration of high-sensitivity C-reactive protein (hs-CRP), interleukin 6 (IL-6), and tumor necrosis factor $\alpha$ (TNF- $\alpha$ ). The inflammatory potential of the diet was assessed using the Dietary Inflammatory Index (DII $\left.{ }^{\circledR}\right)$. Intervention with an anti-inflammatory diet resulted in a significant reduction in body weight and visceral adipose tissue and caused improvements in the participants' cardiometabolic and inflammatory statuses. The anti-inflammatory diet was shown to be effective regarding obesity management. The study data could advance current scientific knowledge in the field of inflammation and diet, provide guidelines for obesity management, and find its application in routine clinical practice.
\end{abstract}

Keywords: anti-inflammatory; cytokines; diet; inflammation; obesity; weight reduction programs

\section{Introduction}

Obesity is a key modifiable risk factor that increases the burden of non-communicable diseases (NCDs), such as type 2 diabetes (T2D), cardiovascular disease (CVD), and cancer, which makes it 
one of the greatest clinical and public health challenges of the 21st century [1]. The current obesity prevalence in most European countries is around 20\% [2], without a systematic difference concerning gender [3]. The association between obesity and NCDs relies on the theory of chronic, low-grade inflammation. Serum levels of inflammatory markers, such as C-reactive protein (CRP), tumor necrosis factor $\alpha$ (TNF- $\alpha$ ), and interleukin 6 (IL-6), correlate with body mass index across the broad range of obesity [4]. The effects of the inflammatory markers that are triggered by excessive adipose tissue have been reported on insulin signaling pathways, resulting in insulin resistance, endothelial dysfunction, and further activation of the innate immune system, eventually progressing to T2D and other cardiometabolic disorders [4-7]. A reduction in excessive body fat mass, particularly visceral fat, has been shown to decrease obesity-induced inflammation, thus leading to the amelioration of obesity-related comorbidities [8,9].

There is a growing body of evidence linking diet with the immune system functioning and the modulation of the inflammatory response [10,11]. An unhealthy diet that is high in saturated fats and refined sugars seems to be one of the main factors implicated in the development of obesity and other NCDs [10], while a diet rich in fruit, vegetables, extra virgin olive oil, legumes, whole grains, fish, nuts, cocoa, coffee, tea, and red wine, similar to the Mediterranean diet, has demonstrated beneficial effects on cardiometabolic risk reduction [12]. The abovementioned food items contain various bioactive components that exhibit anti-inflammatory activity $[12,13]$, and if consumed as a dietary pattern, it could be considered an anti-inflammatory diet, although currently, there is still no single, comprehensive, universally accepted definition of such a diet. Of all dietary patterns, the Mediterranean diet has been shown to have the highest anti-inflammatory potential [14], with beneficial effects on weight regulation, inflammation, and obesity-related cardiometabolic derangements [15-20]. A recently published umbrella review of meta-analyses of randomized controlled trials on the impact of popular dietary patterns on anthropometric and cardiometabolic parameters concluded that among all the diets evaluated, the Mediterranean diet had the strongest and most consistent evidence of an improvement in weight, body mass index (BMI), and cardiometabolic risk factors [21].

\section{Materials and Methods}

The concept of an energy-restricted anti-inflammatory diet for obesity management among younger adults was protocoled as a randomized clinical trial to evaluate its applicability and efficacy [22]. The study was registered with clinicaltrials.gov: NCT03987776. Herein, we report its preliminary results.

\subsection{Subjects}

The participants were recruited during their first visit to the obesity outpatient clinic at the Clinical Hospital Centre Rijeka, Croatia. The inclusion criteria were as follows: participants of both genders, ages $18-50$ years, BMI $\geq 30 \mathrm{~kg} / \mathrm{m}^{2}$ with or without obesity-related complications, and stable body weight for the previous three months.

Exclusion criteria were cigarette smoking within 6 months before the study's initiation; chronic heart, kidney, and/or severe liver disease; malignant disease or history of malignant disease; use of anti-inflammatory or immunosuppressive drugs or medications for weight loss; changes in chronic medications; active infection or surgical procedure in the previous three months; food allergy or intolerance to any anti-inflammatory diet constituent; pregnancy and/or lactation.

The presence of metabolic syndrome was assessed according to the definition from the International Diabetes Federation Task Force on Epidemiology and Prevention [23].

\subsection{Study Design}

This six-month, two-arm randomized controlled trial with a two (group) by two (time) repeated measures design was conducted between March and October 2019 at Clinical Hospital Centre Rijeka, Croatia. Following the study presentation and baseline assessments, the recruited patients were randomly allocated to the anti-inflammatory diet (AID) group or the control diet (CD) group using 
a web-based randomization system (https://www.random.org/), which was run by trained medical personnel who were not engaged in any other study procedure. The study was approved by the ethics committee of the Clinical Hospital Centre Rijeka (Reg. No: 2170-29-02/15-16-4, 31 January 2017) and was conducted in line with the Declaration of Helsinki. Written informed consent was obtained from all participants before they participated in the study.

\subsection{Intervention}

All study participants attended monthly educational workshops that were managed by a clinical dietitian. The AID group participants were strongly encouraged to follow an energy-restricted diet, based on low glycemic foods, whole-grain products, legumes, colorful vegetables and fruits, nuts, seeds, marine fish, olive oil, green/black tea, and multiple spices and herbs. The CD group participants were strongly encouraged to follow an isocaloric standard diet protocol for bodyweight reduction (55-60\% carbohydrates, $25-30 \%$ fat, $15-20 \%$ protein) [24]. A detailed description of the dietary interventions was provided in a study protocol [22]. An overlap in the recommended daily intake of vegetables, fruits, legumes, whole grains, nuts, green tea, and herbs between the AID and CD groups was bridged with more frequent use of olive oil, colorful low glycemic index vegetables and fruits, nuts, seeds, onion, garlic, various spices, marine fish, fermented dairy products, and with the avoidance of red and processed meat and industrially processed foods in AID group.

The daily resting energy expenditure was calculated for each participant according to Mifflin-St. Jeor's equation [25] using their baseline anthropometric measurements, which was then multiplied by the activity factor based on information from the physical activity questionnaire. The value obtained from these equations was reduced by $25 \%$, thus providing the recommended energy intake for each participant. The adjustments of the number and quantity of servings of each food group were made accordingly.

During the workshops, meal planning with recipes, food serving sizes, the consumption of specific foods, and personal goal-setting were discussed. Participants who had missed the educational workshop were provided with workshop materials. If diagnosed with vitamin $\mathrm{D}$ deficiency according to the baseline serum vitamin D status, the participants were recommended to take vitamin $D$ supplements. The intake of other dietary supplements during the trial was noted and included in all dietary calculations.

To assess the compliance with given dietary recommendations, participants were asked to fulfill a 3-day food intake record (covering two weekdays and one weekend day) before a monthly group meeting (overall six 3-day food intake records). After being evaluated, the dietary records were discussed with each participant. Participants whose adherence to a dietary intervention was less than $75 \%$ were considered as non-compliant and were discontinued from the trial.

\subsection{Anthropometry and Body Composition}

The anthropometric measurements were taken using a calibrated integrated weighing platform (seca mBCA 515, secaGmbH\&Co. KG, Hamburg, Germany), with an accuracy of $\pm 50 \mathrm{~g}$ up to $100 \mathrm{~kg}$ and a digital stationary stadiometer with the wireless transmission (seca 264, secaGmbH\&Co. KG, Hamburg, Germany), with an accuracy of $\pm 2 \mathrm{~mm}$. The BMI was calculated as the weight (in kilograms) divided by the square of the height (in meters). Waist circumference (WC) was measured midway between the lowest rib and the upper border of the iliac crest in the medial axillary line at the end of a normal exhalation using a non-stretchable measuring tape (seca 201, secaGmbH\&Co. KG, Hamburg, Germany). The body composition was measured via the bioelectrical impedance method using the measuring platform (seca mBCA 515, secaGmbH\&Co. KG, Hamburg, Germany) with four pairs of electrodes, where one was placed on each arm and foot during the measurement. The impedance was measured with a current of $100 \mu \mathrm{A}$ at frequencies between 1 and $1000 \mathrm{kHz}$. The duration of each bioelectrical impedance measurement was $75 \mathrm{~s}$. Participants were measured in a standing position 
with hands outstretched. All participants were advised not to exercise for $12 \mathrm{~h}$ and not to drink alcohol for $24 \mathrm{~h}$ before measuring the impedance.

\subsection{Cardiometabolic Parameters}

The cardiometabolic parameters included fasting plasma glucose, $\mathrm{HbA1c}$, insulin, total cholesterol, low-density lipoprotein cholesterol (LDL-C), high-density lipoprotein cholesterol (HDL-C), and triglycerides, which were measured at the baseline and the end of the trial. Whole venous blood samples of each participant were collected in the morning by experienced medical personnel. All participants were instructed to fast for at least $12 \mathrm{~h}$ and to restrain from physical activity and alcohol for $24 \mathrm{~h}$ before the blood sampling. Fasting plasma glucose, HbA1c, and blood lipids were analyzed using photometry on an Olympus 5800 (Olympus, Center Valley, PA, USA). The chemiluminescent immunoassay method was used to determine the vitamin D (Cobas E601, Roche, Mannheim, Germany) and insulin concentrations (Immulite 2000xp, Siemens, Eschborn, Germany).

\subsection{Inflammatory Parameters}

To evaluate the inflammatory status, the concentrations of high-sensitivity C-reactive protein (hs-CRP), IL-6, and TNF- $\alpha$ were determined. hs-CRP was assessed using the immunoturbidimetry method on an Olympus 5800 (Olympus, Center Valley, PA, USA), while ELISA assay kits were purchased from eBioscience ${ }^{\mathrm{TM}}$ (Thermo Fisher Scientific, Waltham, MA, USA) and used for the measurement of IL-6 and TNF- $\alpha$. Standard curves were constructed for the determination of each cytokine concentration according to the manufacturers' instructions.

\subsection{The Inflammatory Potential of the Diet}

The inflammatory potential of each participant's diet was assessed with the dietary inflammatory index $\left(\mathrm{DII}{ }^{\circledR}\right)$ [26], which is based on the data from the food frequency questionnaire (FFQ), which has already been applied to the Croatian population [27], together with the data obtained from the 3-day food intake diaries recorded each month of the study, as previously described. The FFQ was recorded at baseline and at the end of the trial to obtain the frequency (from once per month to a few times per day) and portion size (small, medium, and large) information about food and beverage consumption. In addition to the standard list of 97 food items, for this trial, an additional 36 food items and 14 herbs and spices with anti-inflammatory properties were included in the FFQ.

To calculate the energy and dietary components intake, the Croatian food composition database [28] was used. The intake of certain nutrients, such as caffeine, $\beta$-carotene, omega- 3 , and omega- 6 fatty acids, was estimated using the Danish [29] and American food composition database [30], the Phenol-Explorer 3.0 database [31], and the United States Department of Agriculture (USDA) [32]. As thermal processing, including domestic cooking, has long been known to influence the contents of the various polyphenols present in certain food [33], the total polyphenols were multiplied by their retention factors. Polyphenols losses due to boiling were calculated with a retention factor of 0.59 , with 0.56 for frying, and 0.50 for baking [34].

For the DII ${ }^{\circledR}$ calculation, the provided energy and dietary data from the FFQ or 3-day food diary of each participant were first linked to the global means and standard deviations of the food and nutrients intakes from 11 nations to calculate the z-scores [26], then converted to a percentile and centered to minimize the "right skew" by doubling the value and subtracting 1 . The provided percentile score of each food parameter was then multiplied by the respective inflammatory effect score to provide the food parameter-specific DII ${ }^{\circledR}$ score [26]. The overall DII ${ }^{\circledR}$ score for each participant was the summation of forty-five food parameter-specific DII ${ }^{\circledR}$ scores [26]. The positive values of the DII ${ }^{\circledR}$ score indicated a pro-inflammatory diet, and negative values indicated an anti-inflammatory diet [26]. 


\subsection{The Outcomes}

To examine the efficacy of an energy-restricted anti-inflammatory diet in the management of obesity, the changes in body weight, body composition, cardiometabolic (fasting blood glucose, $\mathrm{HbA1c}$, insulin resistance, lipid profile, cardiometabolic index) and inflammatory (hs-CRP, IL-6, TNF- $\alpha$ ) parameters following a 6-month dietary intervention were assessed.

Insulin resistance was calculated using the Homeostatic Model Assessment for Insulin Resistance (HOMA-IR) [35], according to the formula: HOMA-IR $=($ insulin $(\mathrm{mU} / \mathrm{L}) \times$ glucose $(\mathrm{mmol} / \mathrm{L})) / 22.5$. The cardiometabolic index (CMI) was used to assess the presence of diabetes risk and the progression of atherosclerosis as an indicator of visceral adipose tissue distribution and dysfunction according to the formula: $\mathrm{CMI}=($ triglycerides $(\mathrm{mmol} / \mathrm{L}) /$ high-density lipoproteins $(\mathrm{mmol} / \mathrm{L})) \times($ waist circumference $(\mathrm{cm}) /$ body height $(\mathrm{cm}))$ [36]. All changes were calculated using z-scores ((mean after intervention baseline mean)/baseline mean) $\times 100$.

\subsection{Statistical Analysis}

For the purpose of this pilot study, the required sample size was calculated using MedCalc (MedCalc for Windows, version 15.0, MedCalc Software, Ostend, Belgium) based on the changes in hs-CRP levels observed in participants included in an energy-restricted Mediterranean-inspired low glycemic load AI dietary intervention [37]. To detect a meaningful difference in the level of hs-CRP, with an $\alpha$ level of 0.05 , power equal to 0.8 , group size ratio $1: 1$, and using the $t$-test for repeated measures, it was calculated that 33 participants per group were required. The independent $t$-test, Mann-Whitney $U$ test, and $\chi^{2}$ test were used to compare the study groups' characteristics at baseline and week 24 . To test the differences in all participants' characteristics obtained within the study, the $t$-test and Wilcoxon test, ANOVA, and Friedman's ANOVA test for repeated measurements were used. Linear regression analysis was used to evaluate the potential relationships and correlations between the inflammatory potential of the diet as an independent variable with anthropometric, metabolic, and inflammatory variables as dependent variables. Adjustments were made for age, sex, educational level, physical activity, and obesity degree. Models were not adjusted for the total energy intake because it is one of the DII ${ }^{\circledR}$ components. All tests were run with Statistica 12.7 for Windows (Statsoft Inc, Tulsa, OK, USA), which were regarded as two-tailed, and $p$-values $<0.05$ were considered to be statistically significant.

\section{Results}

\subsection{Study Participants}

The flow of participants throughout the study is shown in Figure 1. Out of 125 participants fulfilling the inclusion and exclusion criteria, 63 were randomized to the AID group and 62 into the CD group. Overall, 44 participants were excluded from the study mainly due to non-compliance with the dietary recommendations. Therefore, 81 participants (42 (51.9\%) in the AID group and $39(48.1 \%)$ in the CD group) completed this 6-month study. 


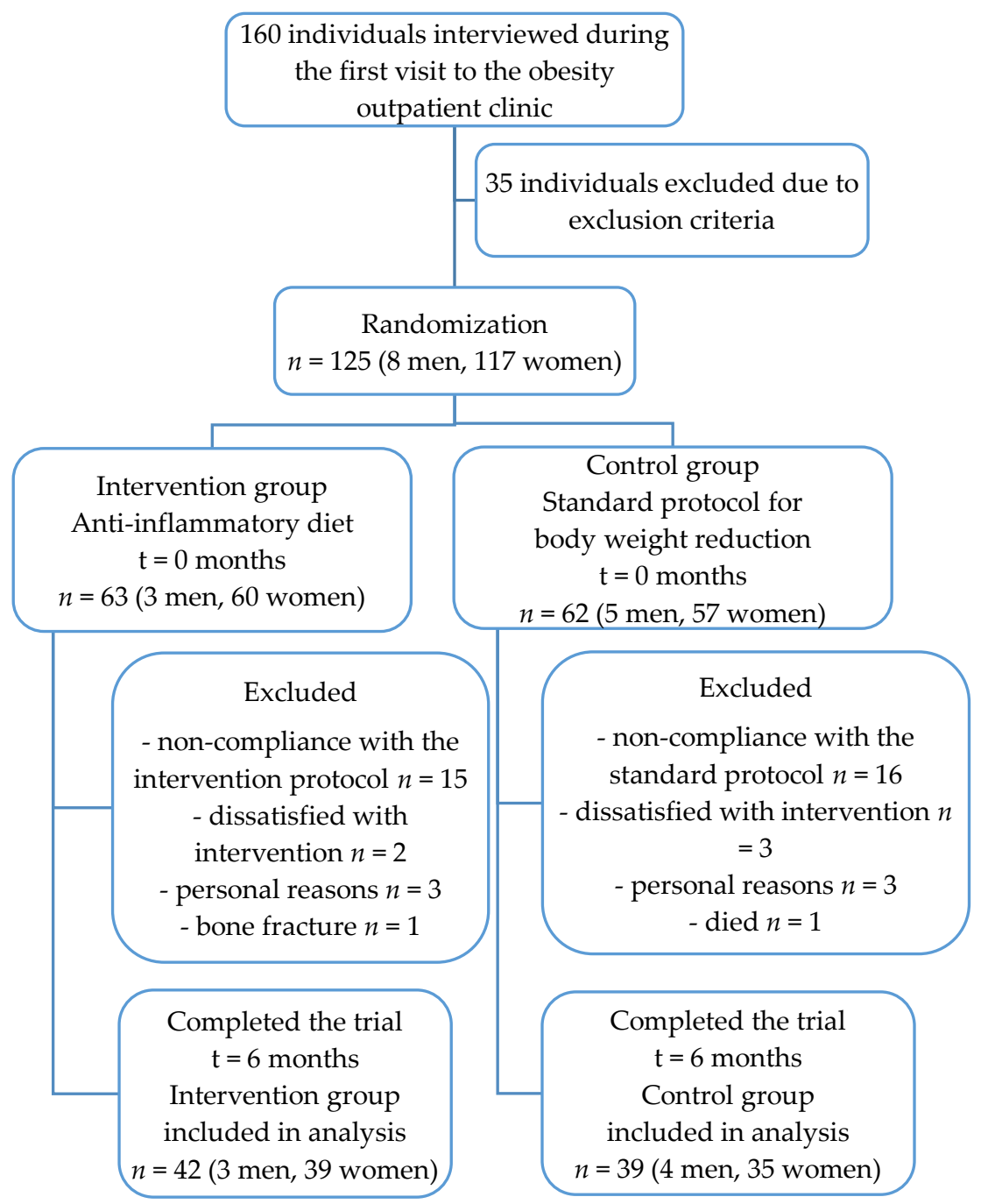

Figure 1. The flow diagram of participants included in the study.

Concerning gender, there was no statistically significant difference between the studied groups $(p=0.619)$ but there were significantly more women $(92.9 \%$ and $89.7 \%)$ than men $(7.1 \%$ and $10.3 \%)$ in both the AID and CD groups, respectively $(p<0.001)$ (Table 1$)$. The mean age of the AID group participants was 44 years, and 42 years in the CD group, without a statistically significant difference $(p=0.178)$. There were significantly more married participants $(90.5 \%$ and $84.5 \%)$ in both groupscompared with other relationship statuses. In the AID group, significantly more participants had a university education (61.9\% in AID vs. $56.4 \%$ in CD, $p=0.002$ ) compared with other education levels, and significantly more participants were employed $(95.2 \%, p=0.033)$ than unemployed in the AID group, while in the CD group, almost a quarter of participants were unemployed (23.1\%). Most of the participants in both groups were physically inactive and hadfirst-degree obesity. Almost half of the participants in the AID group met the International Diabetes Federation-criteria for metabolic syndrome along with one-third in the CD group. Additionally, significantly more participants in the AID group were diagnosed with autoimmune thyroid disease ( $23.8 \%$ in AID vs. $7.7 \%$ in CD, $p=0.048)$. 
Table 1. Socio-demographic characteristics of the participants $(n=81)$.

\begin{tabular}{|c|c|c|c|}
\hline Variable & $\begin{array}{c}\text { Anti-inflammatory } \\
\text { Diet (AID) Group } \\
(42(51.9))\end{array}$ & $\begin{array}{c}\text { Control Diet (CD) } \\
\text { Group } \\
(39(48.1))\end{array}$ & $p$-Value \\
\hline Gender, Men & $3(7.1)$ & $4(10.3)$ & \multirow{2}{*}{$0.619^{\mathrm{a}}$} \\
\hline Women & $39(92.9)$ & $35(89.7)$ & \\
\hline Age (years) $($ Mean \pm SD) & $43.60 \pm 5.75$ & $41.72 \pm 6.70$ & $0.178^{b}$ \\
\hline Marriage Status, Single & $3(7.1)$ & $3(7.7)$ & \multirow{4}{*}{$0.537^{\mathrm{a}}$} \\
\hline Married & $38(90.5)$ & $33(84.6)$ & \\
\hline Divorced & $1(2.4)$ & $3(7.7)$ & \\
\hline Widowed & $0(0)$ & $0(0)$ & \\
\hline Education Degree, Elementary school & $0(0)$ & $2(5.1)$ & \multirow{4}{*}{$0.00^{\mathrm{a}}$} \\
\hline High School & $12(28.6)$ & $22(56.4)$ & \\
\hline University & $29(69.0)$ & $15(38.5)$ & \\
\hline Postgraduate & $1(2.4)$ & $0(0)$ & \\
\hline Professional Status, Unemployed & $1(2.4)$ & $9(23.1)$ & \multirow{3}{*}{$0.033^{\mathrm{a}}$} \\
\hline Employed & $40(95.2)$ & $30(76.9)$ & \\
\hline Student & $1(2.4)$ & $0(0)$ & \\
\hline Physical Activity, Inactive & $15(35.7)$ & $18(46.2)$ & \multirow{4}{*}{$0.315^{a}$} \\
\hline Moderately Inactive & $9(21.4)$ & $3(7.7)$ & \\
\hline Moderately Active & $14(33.3)$ & $15(38.5)$ & \\
\hline Active & $4(9.5)$ & $3(7.7)$ & \\
\hline Obesity, First Degree & $26(61.9)$ & $21(53.8)$ & \multirow{3}{*}{$0.584^{\mathrm{a}}$} \\
\hline Second Degree & $10(23.8)$ & $9(23.1)$ & \\
\hline Third Degree & $6(14.3)$ & $9(23.1)$ & \\
\hline Metabolic Syndrome, Yes & $20(47.6)$ & $13(33.3)$ & $0.191^{a}$ \\
\hline Autoimmune Thyroid Disease & $10(23.8)$ & $3(7.7)$ & $0.048^{a}$ \\
\hline
\end{tabular}

Data are presented as number (\%) unless otherwise stated. ${ }^{a} p<0.05$ was tested using the $\chi^{2}$ test for independent samples. ${ }^{b} p<0.05$ was tested with Student's $t$-test for independent samples.

\subsection{Effect of Dietary Intervention on Diet Quality}

The changes in the dietary parameters of the DII ${ }^{\circledR}$ following the 6-month dietary intervention are presented in Table 2. At the baseline, the AID group had significantly higher intakes of omega- 3 fatty acids $(p=0.015)$, beta-carotene $(p=0.002)$, flavonols $(p=0.029)$, garlic $(p<0.001)$, and thyme/oregano $(p=0.009)$, while the CD group had higher intakes of carbohydrates $(p=0.049)$, omega- 6 fatty acids $(p=0.023)$, trans fatty acids $(p=0.007)$, cholesterol $(p=0.004)$, vitamin A $(p=0.008)$, vitamin B6 $(0.023)$, niacin $(p=0.001)$, riboflavin $(p=0.002)$, thiamine $(p=0.002)$, and zinc $(p=0.025)$. Inthe study trial, the AID group participants significantly reduced the intake of pro-inflammatory components, i.e., the intake of energy $(p<0.001)$, carbohydrates $(p<0.001)$, protein $(p=0.009)$, total fat $(p<0.001)$, saturated fatty acids $(p<0.001)$, trans fat $(p<0.001)$, cholesterol $(p=0.030)$, and vitamin B12 $(p=0.012)$. They also reduced the intake of certain anti-inflammatory components, including monounsaturated fatty acids $(p=0.110)$, polyunsaturated fatty acids $(p=0.010), \mathrm{n}-6$ fatty acids $(p<0.001)$, alcohol $(p<0.001)$, vitamin $\mathrm{D}(p=0.012)$, and flavonones $(p<0.001)$. However, they significantly increased the intake of some other anti-inflammatory components, namely, $\mathrm{n}-3$ fatty acids $(p=0.049)$, fiber $(p=0.002)$, vitamin A $(p=0.020)$, vitamin E $(p=0.015)$, vitamin C $(p=0.002)$, beta carotene $(p<0.001)$, folate $(p<0.001)$, flavones $(p<0.001)$, green/black tea $(p=0.011)$, garlic $(p=0.006)$, pepper $(p=0.006)$, turmeric $(p=0.002)$, and thyme/oregano $(p<0.001)$. Due to the anti-inflammatory dietary intervention, the anti-inflammatory potential of the diet in the AID group became almost four times lower than the value at the baseline $(283.02 \%$ increase, $p=0.002)$. 
Table 2. The changes in the dietary parameters of the DII ${ }^{\circledR}$.

\begin{tabular}{|c|c|c|c|c|c|c|c|c|c|c|}
\hline \multirow[b]{2}{*}{ Variable } & \multicolumn{4}{|c|}{ Anti-inflammatory Diet Group } & \multicolumn{4}{|c|}{ Control Diet Group } & \multirow[b]{2}{*}{$\begin{array}{l}\text { Baseline } \\
p \text {-Value } \mathrm{d}\end{array}$} & \multirow[b]{2}{*}{$\begin{array}{l}\text { Trial End } \\
p \text {-Value } \mathrm{e}^{\mathrm{e}}\end{array}$} \\
\hline & Baseline & Trial End & $\begin{array}{c}\text { Change } \\
(\%)\end{array}$ & $p$-Value ${ }^{\mathrm{c}}$ & Baseline & Trial End & $\begin{array}{c}\text { Change } \\
(\%)\end{array}$ & $p$-Value ${ }^{c}$ & & \\
\hline Energy (MJ) & $10.03(2.64)$ & $6.92(0.47)$ & -31.01 & $<0.001$ & $11.21(2.59)$ & $7.63(0.40)$ & -31.94 & $<0.001$ & 0.129 & $<0.001$ \\
\hline Protein $(\mathrm{g})$ & $103.03(31.08)$ & 85.60 (14.42) & -16.92 & $0.009^{\mathrm{a}}$ & $113.70(26.49)$ & $97.13(8.72)$ & -14.57 & $0.001^{\mathrm{a}}$ & $0.211^{\mathrm{a}}$ & $0.002^{\mathrm{a}}$ \\
\hline Carbohydrate (g) & $233.91(77.56)$ & $145.35(31.43)$ & -37.86 & $<0.001^{\mathrm{a}}$ & $280.21(79.75)$ & $172.96(17.32)$ & -38.27 & $<0.001^{\mathrm{a}}$ & $0.049^{\mathrm{a}}$ & $<0.001^{\text {a }}$ \\
\hline Fat $(\mathrm{g})$ & $111.94(27.14)$ & 81.05 (13.91) & -27.60 & $<0.001^{\mathrm{a}}$ & $120.07(30.28)$ & $80.69(9.58)$ & -32.80 & $<0.001^{\mathrm{a}}$ & $0.338^{a}$ & $0.918^{\mathrm{a}}$ \\
\hline MUFA (g) & $44.93(11.90)$ & $39.30(8.69)$ & -12.53 & $0.110^{\mathrm{a}}$ & $43.93(10.30)$ & $32.39(3.68)$ & -26.27 & $<0.001^{\mathrm{a}}$ & $0.759^{\mathrm{a}}$ & $<0.001^{a}$ \\
\hline PUFA (g) & $19.02(5.39)$ & $15.40(3.63)$ & -19.03 & $0.010^{\mathrm{a}}$ & $21.10(4.92)$ & $13.26(1.24)$ & -37.16 & $<0.001^{\mathrm{a}}$ & $0.174^{\mathrm{a}}$ & $0.009^{\mathrm{a}}$ \\
\hline Omega-3 (g) & $1.16(0.68)$ & $1.27(0.54)$ & 9.48 & $0.049^{\mathrm{a}}$ & $0.79(0.21)$ & $0.54(0.15)$ & -31.65 & $<0.001^{\mathrm{a}}$ & $0.015^{\mathrm{a}}$ & $<0.001^{b}$ \\
\hline Omega-6 (g) & $0.73(0.21)$ & $0.47(0.19)$ & -35.62 & $<0.001^{\mathrm{a}}$ & $0.91(0.30)$ & $0.60(0.12)$ & -3.23 & $0.159^{\mathrm{a}}$ & $0.023^{\mathrm{a}}$ & $<0.001^{a}$ \\
\hline Saturated fat (g) & $41.50(14.69)$ & $20.20(3.10)$ & -51.33 & $<0.001^{\mathrm{a}}$ & $49.35(14.67)$ & $28.63(3.56)$ & -41.99 & $<0.001^{\mathrm{a}}$ & $0.074^{\mathrm{a}}$ & $<0.001^{\text {a }}$ \\
\hline Trans fat (g) & $1.67(0.41)$ & $1.32(0.62)$ & -20.96 & $<0.001^{\mathrm{a}}$ & $2.13(0.67)$ & $1.31(0.30)$ & -38.50 & $<0.001^{\mathrm{a}}$ & $0.007^{\mathrm{a}}$ & $0.941^{\mathrm{a}}$ \\
\hline Cholesterol (mg) & $380.81(160.38)$ & $318.51(175.61)$ & -16.36 & $0.030^{\mathrm{a}}$ & $477.55(463.01)$ & $463.01(125.57)$ & -3.04 & 0.643 & $0.004^{\mathrm{a}}$ & $<0.001^{a}$ \\
\hline Fiber $(\mathrm{g})$ & 27.44 (11.30) & $33.90(5.22)$ & 23.54 & $0.002^{a}$ & $25.46(6.69)$ & $28.69(3.95)$ & 12.69 & 0.146 & $0.467^{\mathrm{a}}$ & $<0.001^{a}$ \\
\hline Alcohol (g) & $6.10(12.29)$ & $0.21(1.02)$ & -96.56 & $<0.001^{\mathrm{b}}$ & $3.34(3.87)$ & $2.44(3.33)$ & -26.95 & $0.605^{b}$ & $0.991^{\mathrm{b}}$ & $0.005^{b}$ \\
\hline Caffeine $(\mathrm{g})$ & $315.41(162.30)$ & $239.48(112.49)$ & -24.07 & $0.675^{\mathrm{a}}$ & 218.35 & $202.07(79.48)$ & -7.46 & $0.864^{\mathrm{b}}$ & $0.095^{\mathrm{a}}$ & $0.193^{\mathrm{a}}$ \\
\hline Folate $(\mu \mathrm{g})$ & $433.81(243.26)$ & $539.07(158.45)$ & 47.86 & $<0.001^{\mathrm{a}}$ & $330.28(82.88)$ & $373.58(53.67)$ & 11.27 & $0.042^{\mathrm{a}}$ & $0.055^{\mathrm{a}}$ & $<0.001^{\text {a }}$ \\
\hline Beta carotene $(\mu \mathrm{g})$ & $4.20(2.16)$ & $5.72(1.95)$ & 36.19 & $<0.001^{\mathrm{b}}$ & $3.75(1.57)$ & $3.32(0.71)$ & -11.47 & $<0.001^{\mathrm{a}}$ & $0.002^{b}$ & $0.193^{\mathrm{a}}$ \\
\hline Vitamin A (RE) & $700.87(334.55)$ & $919.41(570.63)$ & 31.18 & $0.020^{\mathrm{a}}$ & $969.03(530.92)$ & $1083.97(540.80)$ & 11.86 & $0.190^{b}$ & $0.008^{a}$ & $0.187^{\mathrm{a}}$ \\
\hline Vitamin B6 (mg) & $2.24(0.90)$ & $2.42(1.16)$ & 0.00 & $0.480^{\mathrm{a}}$ & $2.81(1.31)$ & $2.00(0.70)$ & -28.83 & $0.022^{b}$ & $0.024^{\mathrm{a}}$ & $0.250^{b}$ \\
\hline Vitamin B12 ( $\mu \mathrm{g})$ & $3.14(1.33)$ & $2.46(1.44)$ & -21.66 & $0.012^{\mathrm{a}}$ & $3.18(1.07)$ & $2.30(0.47)$ & -27.67 & $<0.001^{\mathrm{a}}$ & $0.884^{\mathrm{a}}$ & $0.139^{\mathrm{a}}$ \\
\hline Vitamin C (mg) & $204.13(153.16)$ & 287.64 (106.32) & 40.91 & $0.002^{a}$ & $166.32(76.80)$ & $133.05(33.06)$ & -20.00 & $0.007^{\mathrm{a}}$ & $0.287^{\mathrm{a}}$ & $<0.001^{a}$ \\
\hline Vitamin D ( $\mu \mathrm{g})$ & $14.54(22.06)$ & $6.24(17.41)$ & -57.08 & $0.012^{\mathrm{b}}$ & $10.10(11.68)$ & $2.18(0.96)$ & -78.42 & $<0.001^{\mathrm{a}}$ & $0.966^{b}$ & $0.261^{\mathrm{a}}$ \\
\hline Vitamin E (mg) & $22.51(12.98)$ & $26.81(8.19)$ & 19.10 & $0.015^{\mathrm{a}}$ & $16.12(2.97)$ & $12.35(2.44)$ & -23.39 & $<0.0001^{\mathrm{a}}$ & $0.113^{b}$ & $<0.001^{a}$ \\
\hline Iron (mg) & $8.22(2.05)$ & $8.55(2.12)$ & 4.01 & $0.487^{\mathrm{a}}$ & $9.03(2.81)$ & $9.37(1.85)$ & 3.77 & $0.571^{\mathrm{a}}$ & $0.143^{\mathrm{a}}$ & $0.017^{\mathrm{a}}$ \\
\hline Magnesium (mg) & $412.51(132.81)$ & $440.18(80.88)$ & 6.71 & $0.213^{\mathrm{a}}$ & $393.11(88.66)$ & $371.74(36.78)$ & -5.44 & $0.080^{\mathrm{a}}$ & $0.557^{\mathrm{a}}$ & $<0.001^{\mathrm{a}}$ \\
\hline Niacin (mg) & $19.40(6.37)$ & $17.38(3.91)$ & -10.41 & $0.077^{\mathrm{a}}$ & $24.82(8.20)$ & $17.29(1.68)$ & -30.34 & $<0.001^{\mathrm{a}}$ & $0.001^{\mathrm{a}}$ & $0.881^{\mathrm{a}}$ \\
\hline Riboflavin (mg) & $1.60(0.65)$ & $2.01(0.71)$ & 25.63 & $0.089^{a}$ & $2.07(0.70)$ & $2.24(0.31)$ & 8.21 & $0.189^{\mathrm{a}}$ & $0.002^{\mathrm{a}}$ & $0.056^{\mathrm{a}}$ \\
\hline Selenium $(\mu \mathrm{g})$ & $37.42(17.60)$ & $31.76(10.95)$ & -15.13 & $0.467^{\mathrm{a}}$ & $35.86(7.79)$ & $39.61(10.99)$ & 10.46 & $0.309^{\mathrm{a}}$ & $0.696^{\mathrm{a}}$ & $0.018^{a}$ \\
\hline Thiamine (mg) & $1.54(0.51)$ & $1.73(0.39)$ & 12.34 & $0.057^{\mathrm{a}}$ & $1.93(0.58)$ & $1.56(0.25)$ & -19.17 & $0.001^{\mathrm{a}}$ & $0.002^{\mathrm{a}}$ & $0.023^{\mathrm{a}}$ \\
\hline Zinc (mg) & $15.42(4.59)$ & $13.91(3.17)$ & -9.79 & $0.084^{\mathrm{a}}$ & $18.12(4.85)$ & $13.70(1.30)$ & -24.39 & $<0.001^{\mathrm{a}}$ & $0.025^{\mathrm{a}}$ & $0.772^{\mathrm{a}}$ \\
\hline Flavan-3-ol (mg) & $28.81(26.79)$ & $15.83(10.41)$ & -45.05 & $0.056^{\mathrm{b}}$ & 23.3712 .83 & $8.34(2.39)$ & -64.31 & $<0.001^{\text {a }}$ & $0.807^{b}$ & $<0.001^{b}$ \\
\hline Flavones (mg) & $2.90(2.03)$ & $5.58(3.40)$ & 92.41 & $<0.001^{\mathrm{a}}$ & $2.27(1.40)$ & $3.14(1.46)$ & 38.33 & $0.037^{b}$ & $0.366^{\mathrm{b}}$ & $0.002^{\mathrm{a}}$ \\
\hline Flavonols (mg) & $147.41(78.61)$ & $149.40(6.79)$ & 1.35 & $0.856^{\mathrm{a}}$ & $107.57(34.82)$ & $74.19(39.99)$ & -31.03 & $<0.001^{\mathrm{a}}$ & $0.029^{a}$ & $<0.001^{\text {a }}$ \\
\hline Flavonones (mg) & 46.08 (37.87) & 22.96 (22.86) & -50.17 & $<0.001^{\mathrm{a}}$ & 65.59 (74.68) & $3.64(3.98)$ & -94.45 & $<0.001^{\mathrm{a}}$ & $0.856^{\mathrm{b}}$ & $<0.001^{b}$ \\
\hline Anthocyanidins (mg) & $24.25(34.64)$ & $29.97(28.37)$ & 23.59 & $0.406^{\mathrm{a}}$ & $15.54(9.33)$ & $24.30(19.43)$ & 56.37 & $0.606^{\mathrm{a}}$ & $0.873^{b}$ & $0.426^{\mathrm{a}}$ \\
\hline
\end{tabular}


Table 2. Cont.

\begin{tabular}{|c|c|c|c|c|c|c|c|c|c|c|}
\hline \multirow[b]{2}{*}{ Variable } & \multicolumn{4}{|c|}{ Anti-inflammatory Diet Group } & \multicolumn{4}{|c|}{ Control Diet Group } & \multirow[b]{2}{*}{$\begin{array}{c}\text { Baseline } \\
p \text {-Value } \mathrm{d}\end{array}$} & \multirow[b]{2}{*}{$\begin{array}{l}\text { Trial End } \\
p \text {-Value }\end{array}$} \\
\hline & Baseline & Trial End & $\begin{array}{c}\text { Change } \\
(\%)\end{array}$ & $p$-Value ${ }^{c}$ & Baseline & Trial End & $\begin{array}{c}\text { Change } \\
(\%)\end{array}$ & $p$-Value ${ }^{\mathrm{c}}$ & & \\
\hline Isoflavones (mg) & $0.36(0.22)$ & $0.56(0.28)$ & 55.55 & $0.864^{\mathrm{a}}$ & $0.27(0.36)$ & $0.62(0.49)$ & 129.63 & $0.787^{a}$ & $0.816^{\mathrm{a}}$ & $0.374^{\mathrm{a}}$ \\
\hline Eugenol (mg) & $0.03(0.06)$ & $0.04(0.12)$ & 33.33 & $0.499^{b}$ & $0.02(0.02)$ & $0.00(0.01)$ & -100.00 & $0.004^{b}$ & $0.062^{b}$ & $0.262^{b}$ \\
\hline Green/black tea (g) & $1.07(0.91)$ & $2.86(1.66)$ & 167.29 & $0.011^{\mathrm{a}}$ & $0.96(0.24)$ & $2.08(1.84)$ & 116.67 & $0.009^{\mathrm{a}}$ & $0.358^{\mathrm{a}}$ & $0.686^{\mathrm{a}}$ \\
\hline Garlic (g) & $2.10(1.49)$ & $6.60(2.28)$ & 86.57 & $0.006^{\mathrm{b}}$ & $0.75(1.19)$ & $4.32(1.44)$ & 476.00 & $<0.001^{b}$ & $<0.001^{\mathrm{a}}$ & $<0.001^{\mathrm{a}}$ \\
\hline Ginger (g) & $0.15(0.38)$ & $0.19(0.26)$ & 26.67 & $0.098^{\mathrm{b}}$ & $0.06(0.07)$ & $0.00(0.00)$ & -100.00 & $<0.001^{\mathrm{b}}$ & $0.481^{\mathrm{b}}$ & $<0.001^{\mathrm{b}}$ \\
\hline Onion $(\mathrm{g})$ & $14.61(7.03)$ & $13.11(10.63)$ & -10.27 & $0.074^{\mathrm{a}}$ & $17.97(9.01)$ & $10.89(7.89)$ & -39.40 & $0.009^{\mathrm{b}}$ & $0.163^{\mathrm{a}}$ & $0.418^{\mathrm{a}}$ \\
\hline Pepper (g) & $0.67(0.68)$ & $1.25(0.56)$ & 86.57 & $0.006^{b}$ & $0.55(0.54)$ & $0.70(0.23)$ & 27.27 & $0.033^{\mathrm{a}}$ & $0.621^{b}$ & $<0.001^{\mathrm{a}}$ \\
\hline Rosemary (mg) & $0.11(0.17)$ & $0.17(0.29)$ & 54.55 & $0.909^{a}$ & $0.02(0.03)$ & $0.01(0.01)$ & -50.00 & $<0.001^{b}$ & $0.139^{b}$ & $<0.001^{b}$ \\
\hline Saffron $(\mathrm{g})$ & $0.01(0.01)$ & $0.01(0.02)$ & 0.00 & $0.939^{b}$ & $0.00(0.00)$ & $0.01(0.01)$ & 0.00 & $0.999^{\mathrm{b}}$ & $0.917^{b}$ & $0.884^{\mathrm{b}}$ \\
\hline Turmeric (mg) & $0.38(0.94)$ & $1.05(0.69)$ & 176.32 & $0.002^{b}$ & $0.08(0.18)$ & $0.01(0.03)$ & -87.50 & $0.002^{b}$ & $0.220^{b}$ & $<0.001^{b}$ \\
\hline Thyme/oregano (mg) & $0.09(0.11)$ & $0.29(0.16)$ & 222.22 & $<0.001^{a}$ & $0.04(0.08)$ & $0.13(0.06)$ & 225.00 & $0.002^{b}$ & $0.009^{b}$ & $0.001^{\mathrm{b}}$ \\
\hline DII ${ }^{\circledR}$ & $-0.53(2.29)$ & $-2.03(0.97)$ & 283.02 & $0.002^{\mathrm{a}}$ & $-0.23(1.28)$ & $-0.31(1.01)$ & 30.43 & $0.725^{\mathrm{a}}$ & 0.579 a & $<0.001^{\mathrm{a}}$ \\
\hline
\end{tabular}

Data are presented as the mean (SD). ${ }^{a} p<0.05$ was tested using Student's $t$-test for dependent samples. ${ }^{b} p<0.05$ was tested using the Wilcoxon $z$-test for dependent samples. ${ }^{c}$

Comparison within dietary groups (baseline and after 6 months). $d$ Baseline differences between the AID and CD groups. e Differences after 6 months between the AID and CD groups.

PUFA, polyunsaturated fatty acids; MUFA, monounsaturated fatty acids; RE, retinol equivalent;DII ${ }^{\circledR}$, Dietary Inflammatory Index. The CD group participants also significantly reduced the intake of pro-inflammatory components, the intake of energy $(p<0.001)$, carbohydrates $(p<0.001)$, protein $(p=0.002)$, total fat $(p<0.001)$, saturated fatty acids $(p<0.001)$, trans fat $(p<0.001)$, and vitamin $\mathrm{B} 12(p<0.001)$. The intake of certain anti-inflammatory components, such as monounsaturated fatty acids $(p<0.001)$, polyunsaturated fatty acids $(p<0.001)$, $\mathrm{n}-3$ fatty acids $(p<0.001)$, vitamins B6 $(p=0.022)$, vitamin $\mathrm{C}(p=0.007)$, vitamin $\mathrm{D}(p<0.001)$, vitamin $\mathrm{E}(p<0.001)$, niacin $(p<0.001)$, thiamine $(p<0.001)$, zinc $(p<0.001)$, flavan-3-ol $(p<0.001)$, flavonols $(p<0.001)$, flavonones $(p<0.001)$, eugenol $(p=0.004)$, ginger $(p<0.001)$, rosemary $(p<0.001)$, and turmeric $(p<0.001)$, were reduced, while the intake of other anti-inflammatory components, such as folate $(p<0.001)$, flavones $(p=0.037)$, green/black tea $(p=0.009)$, garlic $(p<0.001)$, pepper $(p=0.033)$, and thyme/oregano $(p<0.001)$, were significantly increased. The anti-inflammatory potential of the diet in the $\mathrm{CD}$ group increased bythe end of the trial, although not significantly $(30.43 \%, p=0.725)$. By significantly reducing the intake of grains and products $(-42.56 \%, p<0.001)$, potatoes $(-62.34 \%, p<0.001)$, sweets $(-90.85 \%, p<0.001)$, and juices and sweetened beverages $(-100.00 \%, p<0.001)$, the AID group participants significantly reduced the proportion of total energy intake from carbohydrates $(-8.58 \%, p<0.001)$ (Tables S1 and S3, Supplementary Materials. The shift in consumption toward the higher intake of nuts $(54.25 \%, p<0.001)$, legumes $(47.50 \%, p=0.094)$, and eggs $(27.25 \%, p=0.314)$ significantly raised the proportion of the total energy intake from proteins $(20.16 \%$, $p<0.001)$ (Tables $\mathrm{S} 1$ and S3, Supplementary Materials). Consequently, the proportions of energy intake from mono- $(26.75 \%, p<0.001)$ and polyunsaturated fatty acids $(17.37 \%, p=0.030)$, particularly n-3 fatty acids $(56.82 \%, p<0.001)$, were increased, while those from saturated fats $(-29.46 \%, p<0.001)$ and omega-6 fatty acids $(-3.70 \%, p=0.003)$ were significantly reduced (Table S3, Supplementary Materials). Similar changes were observed among CD group participants, but not in the intake of monounsaturated fats (Tables S1 and S3, Supplementary Materials). 


\subsection{Effects of the Dietary Intervention on Anthropometric and Body Composition Parameters}

The changes in body weight and body composition following the 6-month dietary intervention are presented in Table 3. At the baseline, there were no significant differences regarding the anthropometric and body composition parameters between the two monitored dietary groups. By the study's end, the total body weight, body fat, and visceral fat had decreased significantly in both groups $(-7.06 \%$, $p<0.001$ vs. $-6.21 \%, p<0.001 ;-12.15 \%, p<0.001$ vs. $-14.10 \%, p<0.001$ and $-22.29 \%, p<0.001$ vs. $-25.43 \%$, respectively). Furthermore, the reduction in skeletal muscle mass was also prominent and practically the same in both groups $(-4.34 \%, p=0.022$ vs. $-4.38 \%, p=0.005)$. Although at the study baseline, an almost equal distribution of BMI categories was observed in the AID and CD groups, by the study's end, $21.4 \%$ of the AID group and $25.6 \%$ of the CD group participants were distributed into the overweight class, while the number of participants with severe obesity reduced by half in the AID group (14.3\% to 7.1\%) and by two thirds in the CD group (23.1\% to 7.7\%) (Table S2, Supplementary Materials).

\subsection{Effect of the Dietary Interventions on the Cardiometabolic and Inflammatory Statuses}

At the baseline, the CD group participants had significantly higher values of $\mathrm{HbA} 1 \mathrm{c}(p=0.071)$, total cholesterol $(p=0.028)$, LDL-C $(p=0.031)$, triglycerides $(p=0.008)$, CMI index $(p=0.016)$, IL-6 ( $p<0.001)$, and TNF- $\alpha(p=0.001)$ compared to the AID group (Table 4$)$. After the 6 months of the trial, in the CD group, a significant reduction in fasting plasma glucose $(-13.08 \%, p<0.001)$, insulin resistance that was assessed using the HOMA-IR index $(-36.34 \%, p=0.002)$, total cholesterol $(-7.71 \%, p=0.002)$, and LDL-C $(-11.98 \%, p=0.001)$ was observed, while the status of glycated hemoglobin remained almost the same (Table 4). The AID group participants increased their HDL-C concentrations more than the CD group participants $(10.22 \%, p=0.058$ vs. $-0.75 \%, p=0.073)$. The CMI index decreased in both study groups but not significantly $(-22.73 \%, p=0.153 \mathrm{vs} .-16.90 \%, p=0.622)$.

At the baseline, almost half of the participants in the AID group met the IDF criteria for metabolic syndrome and one-third in the CD group, and following the 6-month dietary intervention, the number of participants fulfilling the criteria for metabolic syndrome decreased by one-third in both groups (Table S2 Supplementary Materials).

Compared with the baseline, the AID group participants significantly increased their serum vitamin D concentration $(38.35 \%, p<0.001)$, while the CD group participants reduced its level, but not significantly $(-10.61 \%, p=0.135)$ (Table 4$)$. The biomarkers of inflammation were significantly reduced in both groups (Table 4). The CD group participants reduced their hs-CRP $(29.46 \%, p=0.003$ vs. $-42.18 \%, p=0.010)$ and IL-6 $(-18.18 \%, p=0.013$ vs. $-26.86 \%, p=0.002)$ concentrations slightly more than the AID group participants, while the AID group participants achieved a greater reduction in TNF- $\alpha(-34.21 \%, p=0.002$ vs. $-10.47 \%, p<0.001)$.

\subsection{Associations Between the Inflammatory Potential of the Diet and the Anthropometric, Cardiometabolic,} and Inflammatory Parameters

The linear regression analysis of the participants' anthropometric, cardiometabolic, and inflammatory parameters after 6 months of the dietary intervention as dependent variables and changes in DII ${ }^{\circledR}$ scores as an independent variable (Table 5) showed a statistically significant negative association only with IL-6 ( $\beta=-0.46,95 \% \mathrm{CI}=-0.56$ to $0.46, p=0.026)$ among the AID group participants. Among the CD group participants, a statistically significant negative association with insulin $(\beta=-0.55,95 \% \mathrm{CI}=-0.92$ to $-0.18, p=0.005)$, HOMA-IR $(\beta=-0.49,95 \% \mathrm{CI}=-0.88$ to $-0.11, p=0015)$, triglycerides $(\beta=-0.56,95 \% \mathrm{CI}=-0.93$ to $-0.20, p=0.004)$, and the CMI index $(\beta=-0.59,95 \% \mathrm{CI}=-0.94$ to $-0.23, p=0.003)$ were observed. There were no other statistically significant associations. 
Table 3. The changes in the anthropometric and body composition parameters.

\begin{tabular}{|c|c|c|c|c|c|c|c|c|c|c|}
\hline \multirow[b]{2}{*}{ Variable } & \multicolumn{4}{|c|}{ Anti-inflammatory Diet Group } & \multicolumn{4}{|c|}{ Control Diet Group } & \multirow[b]{2}{*}{$\begin{array}{l}\text { Baseline } \\
p \text {-Value } \mathrm{d}\end{array}$} & \multirow[b]{2}{*}{$\begin{array}{l}\text { Trial End } \\
p \text {-Value }\end{array}$} \\
\hline & Baseline & Trial End & $\begin{array}{c}\text { Change } \\
(\%)\end{array}$ & $p$-Value ${ }^{\mathrm{c}}$ & Baseline & Trial End & $\begin{array}{c}\text { Change } \\
(\%)\end{array}$ & $p$-Value ${ }^{\mathrm{c}}$ & & \\
\hline Body weight $(\mathrm{kg})$ & $102.94(14.19)$ & 95.67 (11.72) & -7.06 & $<0.001^{\mathrm{a}}$ & $101.35(21.93)$ & $95.06(21.36)$ & -6.21 & $<0.001^{\mathrm{a}}$ & $0.770^{\mathrm{a}}$ & $0.903^{a}$ \\
\hline Body mass index $\left(\mathrm{kg} / \mathrm{m}^{2}\right)$ & $35.36(4.27)$ & $32.90(3.90)$ & -6.96 & $<0.001^{\mathrm{a}}$ & $33.40(5.48)$ & $30.99(4.32)$ & -7.22 & $<0.001^{\mathrm{a}}$ & $0.179^{\text {a }}$ & $0.119^{\mathrm{a}}$ \\
\hline Waist circumference $(\mathrm{cm})$ & $108.43(8.37)$ & $102.91(7.77)$ & -5.09 & $<0.001^{\mathrm{a}}$ & $107.86(10.11)$ & $100.88(10.04)$ & -6.47 & $<0.001^{\mathrm{a}}$ & $0.482^{b}$ & $0.442^{\mathrm{a}}$ \\
\hline Fat tissue $(\mathrm{kg})$ & 46.49 (10.17) & $40.84(7.87)$ & -12.15 & $<0.001^{a}$ & $45.89(7.71)$ & $39.42(8.82)$ & -14.10 & $<0.001^{\mathrm{a}}$ & $0.807^{b}$ & $0.328^{b}$ \\
\hline Fat tissue $(\%)$ & $44.87(4.38)$ & $42.34(4.80)$ & -5.64 & $<0.001^{\mathrm{a}}$ & $45.57(2.57)$ & $42.20(2.98)$ & -7.40 & $0.001^{\mathrm{a}}$ & $0.505^{\mathrm{a}}$ & $0.755^{\mathrm{a}}$ \\
\hline Visceral adipose tissue (l) & $3.14(1.32)$ & $2.44(1.05)$ & -22.29 & $<0.001^{a}$ & $3.46(1.63)$ & $2.58(1.37)$ & -25.43 & $<0.001^{\mathrm{a}}$ & $0.376^{\mathrm{b}}$ & $0.798^{b}$ \\
\hline Non-fat tissue $(\mathrm{kg})$ & $56.45(6.50)$ & $55.28(6.09)$ & -2.07 & $0.139^{a}$ & $54.88(11.50)$ & $53.81(12.97)$ & -1.95 & $0.019^{b}$ & $0.569^{b}$ & $0.625^{\mathrm{a}}$ \\
\hline Non-fat tissue $(\%)$ & $55.13(4.38)$ & $57.80(4.66)$ & 4.84 & $<0.001^{\mathrm{a}}$ & $54.40(2.61)$ & $57.21(2.40)$ & 5.17 & $<0.001^{\mathrm{a}}$ & $0.484^{a}$ & $0.587^{\mathrm{a}}$ \\
\hline Skeletal muscle tissue $(\mathrm{kg})$ & $27.41(3.86)$ & $26.22(3.29)$ & -4.34 & $0.022^{\mathrm{a}}$ & $26.96(6.21)$ & $25.78(7.34)$ & -4.38 & $0.005^{b}$ & $0.449^{b}$ & $0.085^{b}$ \\
\hline Total water $(\mathrm{L})$ & $42.40(4.95)$ & $41.33(4.40)$ & -2.52 & $0.009^{a}$ & $41.16(8.26)$ & $40.04(9.50)$ & -2.72 & $0.091^{\mathrm{a}}$ & $0.537^{\mathrm{a}}$ & $0.053^{\mathrm{b}}$ \\
\hline Total water (\%) & $41.13(8.37)$ & 43.17 (4.08) & 4.96 & $<0.001^{a}$ & $40.34(1.70)$ & $42.66(1.81)$ & 5.73 & $0.009^{a}$ & $0.407^{\mathrm{a}}$ & $0.582^{\mathrm{a}}$ \\
\hline
\end{tabular}

Data are presented as the mean (SD). ${ }^{\mathrm{a}} p<0.05$ was tested with Student's $t$-test for dependent samples. ${ }^{\mathrm{b}} p<0.05$ was tested using the Wilcoxon z-test for dependent samples. ${ }^{\mathrm{c}}$ Comparison within dietary groups (baseline and after 6 months). ${ }^{\mathrm{d}}$ Baseline differences between the AID and CD groups. ${ }^{\mathrm{e}}$ Differences after 6 months between the AID and CD groups. 
Table 4. The changes in the cardiometabolic and inflammatory parameters.

\begin{tabular}{|c|c|c|c|c|c|c|c|c|c|c|}
\hline \multirow[b]{2}{*}{ Variable } & \multicolumn{4}{|c|}{ Anti-inflammatory Diet Group } & \multicolumn{4}{|c|}{ Control Diet Group } & \multirow[b]{2}{*}{$\begin{array}{l}\text { Baseline } \\
p \text {-Value } \mathrm{d}\end{array}$} & \multirow[b]{2}{*}{$\begin{array}{l}\text { Trial End } \\
p \text {-Value }{ }^{\mathrm{e}}\end{array}$} \\
\hline & Baseline & Trial End & $\begin{array}{c}\text { Change } \\
(\%)\end{array}$ & $p$-Value ${ }^{\mathrm{c}}$ & Baseline & Trial End & $\begin{array}{c}\text { Change } \\
(\%)\end{array}$ & $p$-Value ${ }^{\mathrm{c}}$ & & \\
\hline Glucose $(\mathrm{mmol} / \mathrm{L})$ & $5.69(1.41)$ & $5.48(0.62)$ & -3.69 & $0.284^{b}$ & $5.58(0.45)$ & $4.85(0.59)$ & -13.08 & $<0.001^{\mathrm{a}}$ & $0.107^{\mathrm{b}}$ & $<0.001^{\mathrm{a}}$ \\
\hline $\mathrm{HbA} 1 \mathrm{c}(\mathrm{mmol} / \mathrm{mol})$ & $35.26(6.45)$ & $34.66(7.62)$ & -1.70 & $0.855^{\mathrm{a}}$ & $38.34(4.87)$ & $38.37(4.74)$ & 0.08 & $0.121^{\mathrm{a}}$ & $0.071^{\mathrm{a}}$ & $0.050^{\mathrm{a}}$ \\
\hline $\mathrm{HbA} 1 \mathrm{c}(\%)$ & $5.40(0.63)$ & $5.52(0.55)$ & 2.22 & $0.049^{\mathrm{a}}$ & $5.64(0.45)$ & $5.65(0.45)$ & 0.18 & $0.127^{\mathrm{a}}$ & $0.128^{a}$ & $0.371^{\mathrm{a}}$ \\
\hline Insulin (mU/L) & $18.22(11.69)$ & $16.19(9.98)$ & -11.14 & $0.946^{\mathrm{a}}$ & $16.10(4.91)$ & $11.73(3.88)$ & -27.14 & $0.008^{b}$ & $0.419^{b}$ & $0.048^{\mathrm{a}}$ \\
\hline HOMA - IR (pmol/L) & $4.84(3.89)$ & $4.09(3.00)$ & -15.50 & $0.307^{b}$ & $3.99(1.26)$ & $2.54(0.86)$ & -36.34 & $0.002^{\mathrm{a}}$ & $0.572^{b}$ & $0.040^{\mathrm{b}}$ \\
\hline Total cholesterol $(\mathrm{mmol} / \mathrm{L})$ & $5.25(1.10)$ & $4.97(1.34)$ & -5.33 & $0.594^{\mathrm{a}}$ & $5.84(0.65)$ & $5.39(0.80)$ & -7.71 & $0.002^{\mathrm{a}}$ & $0.028^{a}$ & $0.193^{\mathrm{a}}$ \\
\hline HDL-C $(\mathrm{mmol} / \mathrm{L})$ & $1.37(0.21)$ & $1.51(0.53)$ & 10.22 & $0.058^{\mathrm{a}}$ & $1.34(0.20)$ & $1.33(0.13)$ & -0.75 & $0.073^{\mathrm{a}}$ & $0.642^{\mathrm{a}}$ & $0.127^{b}$ \\
\hline LDL-C (mmol/L) & $3.30(1.02)$ & $3.15(0.99)$ & -4.55 & $0.357^{\mathrm{a}}$ & $3.84(0.61)$ & $3.38(0.63)$ & -11.98 & $<0.001^{\mathrm{a}}$ & $0.031^{\mathrm{a}}$ & $0.343^{\mathrm{a}}$ \\
\hline Triglycerides (mmol/L) & $1.31(0.94)$ & $1.15(0.56)$ & -12.21 & $0.446^{\mathrm{b}}$ & $1.50(0.40)$ & $1.33(0.47)$ & -11.33 & $0.393^{\mathrm{a}}$ & $0.008^{b}$ & $0.144^{\mathrm{b}}$ \\
\hline CMI index & $0.66(0.60)$ & $0.51(0.39)$ & -22.73 & $0.153^{b}$ & $0.71(0.21)$ & $0.59(0.22)$ & -16.90 & $0.622^{\mathrm{a}}$ & $0.016^{\mathrm{b}}$ & $0.145^{b}$ \\
\hline Vitamin D (nmol/L) & $48.13(21.36)$ & $66.59(24.72)$ & 38.35 & $<0.001^{\mathrm{a}}$ & $54.09(11.47)$ & $48.35(18.46)$ & -10.61 & $0.135^{\mathrm{b}}$ & $0.237^{\mathrm{a}}$ & $0.006^{\mathrm{a}}$ \\
\hline hs-CRP (mg/L) & $6.28(5.48)$ & $4.43(4.29)$ & -29.46 & $0.003^{\mathrm{a}}$ & $6.78(4.09)$ & $3.92(0.92)$ & -42.18 & $0.010^{b}$ & $0.311^{\mathrm{b}}$ & $0.662^{b}$ \\
\hline IL-6 (pg/mL) & $0.77(0.63)$ & $0.63(0.36)$ & -18.18 & $0.013^{\mathrm{a}}$ & $1.34(0.85)$ & $0.98(0.81)$ & -26.86 & $0.002^{\mathrm{a}}$ & $<0.001^{\mathrm{a}}$ & $0.001^{\mathrm{a}}$ \\
\hline TNF- $\alpha(p g / m L)$ & $0.38(0.19)$ & $0.25(0.09)$ & -34.21 & $0.002^{\mathrm{a}}$ & $1.72(0.33)$ & $1.54(0.36)$ & -10.47 & $<0.001^{\mathrm{a}}$ & $0.001^{\mathrm{b}}$ & $<0.001^{\mathrm{b}}$ \\
\hline
\end{tabular}

Data are presented as the mean (SD). a $p<0.05$ tested with Student's $t$-test for dependent samples. ${ }^{\mathrm{b}} p<0.05$ tested with the Wilcoxon z-test for dependent samples. ${ }^{\mathrm{c}}$ Comparison
within dietary groups (baseline and after 6 months). ${ }^{\mathrm{d}}$ Baseline differences between the AID and CD groups. ${ }^{\mathrm{e}}$ Differences after 6 months between the AID and CD groups. HbA1c, glycated hemoglobin; HOMA-IR, Homeostatic Model Assessment for Insulin Resistance; HDL-C, high-density lipoprotein cholesterol; LDL-C, low-density lipoprotein cholesterol; CMI, cardiometabolic index; hs-CRP, high-sensitivity C-reactive protein; IL-6, interleukin-6; TNF- $\alpha$, tumor necrosis factor alpha. 
Table 5. Regression analyses of the participants' anthropometric, cardiometabolic, and inflammatory parameters after 6 months of dietary intervention as dependent variables and changes in the DII ${ }^{\circledR}$ scores as independent variables.

\begin{tabular}{|c|c|c|c|c|c|c|c|c|}
\hline \multirow{3}{*}{$\begin{array}{c}\text { Variable } \\
\text { dy weight }(\mathrm{kg})\end{array}$} & \multicolumn{4}{|c|}{ Anti-inflammatory Diet Group } & \multicolumn{4}{|c|}{ Control Diet Group } \\
\hline & \multirow{2}{*}{$\frac{\beta}{-0.13}$} & \multicolumn{2}{|c|}{$95 \%$ CI } & \multirow{2}{*}{$\frac{p \text {-Value }}{0.540}$} & \multirow{2}{*}{$\frac{\beta}{-0.21}$} & \multicolumn{2}{|c|}{$95 \% \mathrm{CI}$} & \multirow{2}{*}{$\frac{p \text {-Value }}{0.328}$} \\
\hline & & -0.31 & 0.58 & & & -0.64 & 0.22 & \\
\hline Body mass index $\left(\mathrm{kg} / \mathrm{m}^{2}\right)$ & -0.19 & -0.25 & 0.69 & 0.247 & -0.33 & -0.75 & 0.09 & 0.114 \\
\hline Waist circumference $(\mathrm{cm})$ & -0.06 & -0.16 & 0.79 & 0.087 & -0.12 & -0.56 & 0.32 & 0.582 \\
\hline Fat mass $(\mathrm{kg})$ & -0.18 & -0.26 & 0.70 & 0.234 & -0.26 & -0.69 & 0.16 & 0.214 \\
\hline Fat free mass (kg) & -0.03 & -0.48 & 0.42 & 0.897 & -0.03 & -0.47 & 0.41 & 0.899 \\
\hline Skeletal muscle tissue (kg) & 0.05 & -0.41 & 0.50 & 0.831 & -0.03 & -0.48 & 0.41 & 0.875 \\
\hline Visceral adipose tissue (L) & -0.21 & -0.23 & 0.65 & 0.339 & -0.11 & -0.33 & 0.55 & 0.608 \\
\hline Total water (L) & -0.00 & -0.46 & 0.45 & 0.990 & -0.04 & -0.48 & 0.40 & 0.853 \\
\hline Glucose (mmol/L) & -0.14 & -0.59 & 0.31 & 0.525 & -0.02 & -0.42 & 0.46 & 0.932 \\
\hline $\mathrm{HbA} 1 \mathrm{c}(\mathrm{mmol} / \mathrm{mol})$ & -0.17 & -0.28 & 0.61 & 0.446 & -0.04 & -0.34 & 0.49 & 0.835 \\
\hline HbA1c (\%) & -0.02 & -0.44 & 0.47 & 0.943 & -0.03 & -0.44 & 0.47 & 0.878 \\
\hline Insulin (mU/L) & -0.04 & -0.49 & 0.42 & 0.865 & -0.55 & -0.92 & -0.18 & 0.005 \\
\hline HOMA-IR (pmol/L) & -0.06 & -0.52 & 0.39 & 0.778 & -0.49 & -0.88 & -0.11 & 0.015 \\
\hline Total cholesterol (mmol/L) & -0.01 & -0.44 & 0.46 & 0.961 & -0.38 & -0.79 & 0.03 & 0.071 \\
\hline HDL-C (mmol/L) & -0.05 & -0.51 & 0.40 & 0.814 & 0.17 & -0.27 & 0.61 & 0.428 \\
\hline LDL-C (mmol/L) & -0.10 & -0.55 & 0.35 & 0.659 & -0.22 & -0.65 & 0.21 & 0.300 \\
\hline Triglycerides (mmol/L) & -0.13 & -0.58 & 0.32 & 0.565 & -0.56 & -0.93 & -0.20 & 0.004 \\
\hline CMI index & -0.05 & -0.51 & 0.40 & 0.804 & -0.59 & -0.94 & -0.23 & 0.003 \\
\hline Vitamin D (nmol/L) & -0.07 & -0.39 & 0.52 & 0.766 & -0.23 & -0.66 & 0.20 & 0.277 \\
\hline hs-CRP (mg/L) & -0.06 & -0.40 & 0.51 & 0.798 & -0.21 & -0.64 & 0.22 & 0.319 \\
\hline IL-6 (pg/mL) & -0.46 & -0.56 & 0.46 & 0.026 & -0.14 & -0.58 & 0.30 & 0.520 \\
\hline TNF- $\alpha(\mathrm{pg} / \mathrm{mL})$ & -0.17 & -0.62 & 0.28 & 0.440 & -0.05 & -0.50 & 0.39 & 0.804 \\
\hline
\end{tabular}

Models were adjusted for diet (the group of intervention), age, gender, and physical activity. Models were not adjusted for total energy intake because it is one of the DII ${ }^{\circledR}$ components. HbA1c, glycated hemoglobin; HOMA-IR, Homeostatic Model Assessment for Insulin Resistance; HDL-C, high-density lipoprotein cholesterol; LDL-C, low-density lipoprotein cholesterol; CMI, cardiometabolic index; hs-CRP, high-sensitivity C-reactive protein; IL-6, interleukin-6; TNF- $\alpha$, tumor necrosis factor-alpha.

\section{Discussion}

In the present study, the effects of two energy-restricted dietary patterns on the anthropometric, cardiometabolic, and inflammatory parameters of participants with obesity were assessed. Both intervention dietary patterns, described elsewhere [22], had characteristics of the Mediterranean diet, which has been shown to have numerous and significant health benefits, including the prevention of obesity-related diseases [20,38-41], as well as weight regulation [16,42-44]. As the Mediterranean diet has been acknowledged for having anti-inflammatory properties [45], a more frequent and higher intake of foods with anti-inflammatory characteristics was promoted more among the AID group participants. However, a recently published Cochrane systematic review reported that there is still some uncertainty regarding the effects of a Mediterranean-style diet on clinical outcomes and cardiovascular risk factors [46], concluding that the ongoing studies may provide more solid evidence. The study data could advance current scientific knowledge in the field of inflammation and diet, provide guidelines for obesity management, and find its application in routine clinical practice.

\subsection{The Effect of the Dietary Interventions on Diet Quality Changes}

At the study baseline, the average energy intake of the participants in both groups was higher than recommended for their gender, age, and level of physical activity as a result of higher consumption of animal-origin foods and their products, industrially processed cereals, sweets, sweetened beverages, and added fats than recommended, which is in line with Croatian studies on diet quality [27,47-51]. Increased consumption of the above-mentioned food is significantly associated with weight gain, obesity, obesity-related complications, and low-grade chronic inflammation $[10,12]$. The applied diets 
in both groups can be considered to be high-protein diets [52] because they reach or exceed $20 \%$ of the total energy intake. In the short term, a simultaneously high-protein and low-carbohydrate diet is recommended as a method for weight loss [52]. However, because dietary patterns with this macronutrient ratio generally contain a high proportion of animal-origin foods and saturated fats, which have a significant effect on metabolism and intestinal health, they are not recommended for long-term use [52]. Therefore, in this study, the higher protein content was achieved by a higher intake of fish, legumes, low-fat dairy products, and nuts, and by reducing the intake of meat and their products, as well as sweets, the negative impact of saturated fats was mitigated. By increasing the intake of vegetables and adapting the intake of fruits, participants achieved a change in carbohydrate and fiber intake that is aligned with the characteristics of the Mediterranean and anti-inflammatory diets. Using the DII ${ }^{\circledR}$ to assess the inflammatory potential of the diet, the average diet of both groups at baseline had a minor anti-inflammatory potential due to the consumption of fruits, vegetables, and fish within the recommended intake. These foods contain nutrients that are associated with a significant reduction in low-grade inflammation [10,12]. During the trial, the AID group participants significantly reduced the intake of grains and their products, milk and dairy products, meat and their products, potatoes, sweets and desserts, and alcoholic and non-alcoholic sweetened beverages, which enabled the significant reduction in energy intake, saturated fats, cholesterol, and carbohydrates, which are all acknowledged as being pro-inflammatory [26]. The CD group also made significant reductions in the above-mentioned food items but in minor quantities.

All participants significantly increased their intake of total flavonoids but significantly reduced the intake of flavan-3-ol and flavonols due to their reduced consumption of certain types of fruits, beans, fruit juices, teas, and desserts rich in cocoa. By significantly raising the intake of green leafy and cruciferous vegetables, green tea, and various herbs and spices, and changing the fruit types, the AID group participants significantly raised the intake of beta-carotene and various phenolic substances, especially the intake of flavone. Higher consumption of food, herbs, and spices expressing antioxidant and anti-inflammatory properties may have preventive and/or therapeutic effects on cardiovascular diseases, neurodegenerative disorders, cancer, and obesity [53-55]. The CD group participants also significantly increased the intake of vegetables, spices, and herbs that are typical of the Mediterranean diet, and reduced the intake of fruits, which significantly increased the intake of flavones and other phenolic substances, such as stilbenes and lignans. So far, the intake of various phenolic substances has not been investigated in Croatia, except for flavonoids intake in a cross-sectional study conducted in the same part of Croatia as the present study [27]; thus, this study provides valuable information on the average intake of phenolic substances at both the baseline and the trial's end. Compared to the mentioned Croatian study [27], the baseline average intake of all phenolic substances by both dietary groups was higher than its average intake in the abovementioned Croatian study.

Furthermore, this study is unique in Croatia by providing the average intake data of different spices and herbs, because so far, no data on their intake in Croatia have been recorded. The European population consumes on average about $0.5 \mathrm{~g}$ of spices per day [56,57]. The intake of herbs and spices is thought to have positive health effects toward preventing or alleviating chronic diseases, such as cardiovascular disease, cancer, arthritis, and neurodegenerative disorders, due to the high content of phenolic substances or their metabolites, which all act on specific receptors or enzymes involved in various anti-inflammatory pathways or immune responses [58]. At the study baseline, the AID group participants consumed almost twice as many spices as the CD group participants, which is three times higher than the average of the European population. At the end of the study, both groups consumed all spices and herbs around three times more than at baseline, especially garlic and Mediterranean spices. The AID group participants doubled their intake of pepper and tripled their intake of turmeric, while at the end of the study, the consumption of turmeric was almost twice as much as the global average [26]. The CD group participants significantly increased their intake of pepper and decreased their intake of turmeric. Since curcumin, the active substance in turmeric, has very low oral bioavailability due to its poor solubility, low absorption, rapid metabolism, and systemic elimination [59], the simultaneous 
intake of pepper and turmeric can increase its bioavailability by $154 \%$ [60]. Turmeric, or curcumin, has been very well researched due to its many favorable biological and health effects, including antioxidant, anti-inflammatory, and anti-tumor activity [59]. Furthermore, curcumin may have a potential benefit in weight management, as it inhibits angiogenesis, adipogenesis, and reduces the fat accumulation in mature adipocytes in a mouse model, with a consequent reduction in adipose tissue and weight gain in mice [59]. The AID group participants significantly increased the anti-inflammatory potential of their diet by increasing the intake of garlic and turmeric, as did the CD group participants by increasing their intake of garlic and pepper.

At the baseline, both groups had a diet with mild anti-inflammatory potential due to higher consumption of foods containing nutrients with anti-inflammatory effects, namely, fruits, vegetables, and fish. One-third of all participants had an average diet with pro-inflammatory potential, which was significantly reduced by the change in diet quality, which was mainly due to reduced energy and macronutrient intake, and the significant increase in intake of nutrients with anti-inflammatory potential. Most studies that have used the change in DII ${ }^{\circledR}$ as a tool to assess the inflammatory potential of a diet were observational or retrospective, and till now, there is limited evidence from intervention trials to support the claim that a reduction in DII ${ }^{\circledR}$ scores through dietary changes leads to improvements regarding inflammation or related health risk factors [61-64]. The change in the anti-inflammatory potential of the diet in the AID group toward higher anti-inflammatory potential was somewhat smaller compared to similar studies $[63,65]$. The greater change in DII ${ }^{\circledR}$ observed in these studies may reflect the higher pro-inflammatory potential of the diet at baseline in comparison to our study. However, our study outcomes showed comparable changes relative to the mentioned studies. The reduction in the DII ${ }^{\circledR}$ scores achieved by the AID group was similar to that reported by an Australian study [62]. The authors of another study evaluating the impact of a 12-month inflammation management intervention on the $\mathrm{DII}{ }^{\circledR}$, inflammation, and lipids explained the similar changes in DII ${ }^{\circledR}$ as being due to the reduced number of contacts with study participants, and therefore a lower degree of compliance with dietary recommendations [63]. In our study, a rather mild change in the anti-inflammatory potential of the diet could be explained in terms of a moderate change in the quantity of the nutrients with ahigher anti-inflammatory potential as part of the baseline diet.

\subsection{The Effect of the Dietary Interventions on the Anthropometric and Body Composition Parameters}

As expected, in the present study, the energy-reduced dietary interventions led to significant weight reduction and body composition changes in both groups. Both groups similarly, and significantly, compared to baseline, decreased their weight, BMI, waist circumference, the amount of total and visceral adipose tissue, and skeletal muscle mass. Most of the CD group participants reduced their weight by up to $5 \%$, while most of the AID group participants reduced their weight in the range of 6 to $10 \%$. On average, all participants in this study reduced their weight and BMI by about $7 \%$, which was slightly better than a similar six-month Croatian weight management study, where participants lost on average $10 \%$ of their initial body weight and $4 \%$ of their BMI [65]. However, the aforementioned study included more participants, and besides a low-fat dietary intervention ( $<30 \%$ of total fats) and pharmacological treatment with orlistat, the participants were encouraged to increase their level of physical activity. Furthermore, an average reduction in body weight, waist circumference, and BMI observed in both treatment arms of the present study was more pronounced than that reported in the five controlled clinical trials that evaluated the Mediterranean diet for obesity management [16]. In comparison to the results of a weight regulation study for the control of polycystic ovary syndrome that used a Mediterranean-inspired low glycemic load anti-inflammatory diet [37] while engaging in physical activity, our participants achieved very similar changes in the body composition. However, the visceral fat mass reduction was greater in our study, while the total fat mass reduction was more noticeable in the aforementioned study [37]. Nevertheless, it is important to underline that, unlike the other weight management studies, our participants were counseled not to engage in additional physical 
activity to facilitate the interpretation of the effect of the applied diet on the observed changes in body weight and composition.

\subsection{Effect of the Dietary Interventions on the Cardiometabolic and Inflammatory Statuses}

A recent review showed that moderate weight loss (between 5 and $10 \%$ of one's initial body weight) resulted in a significant reduction in glucose, triglycerides, and total cholesterol and improved insulin sensitivity by between 30 and $60 \%$, which are effects greater than those seen with insulin-sensitizing drugs [66]. In the CD group, a significant reduction in fasting plasma glucose, insulin resistance that was assessed using the HOMA-IR index, and total cholesterol and LDL-C was observed, while the level of $\mathrm{HbA} 1 \mathrm{c}$ remained almost the same. On the other hand, the AID group participants significantly increased their HDL-C, while the reductions in their fasting plasma glucose, HbA1c, HOMA-IR index, total cholesterol, and LDL-C were not significant. These results may be explained at least in part by the fact that the participants in the AID group had slightly higher levels of fasting glucose, insulin, HOMA-IR index, and $\mathrm{HbA} 1 \mathrm{c}$ at baseline than the participants in the CD group. Although $\mathrm{HbA1c}$ remains the standard clinical marker of glycemic control, glycemic variability (GV) is an emerging target for blood glucose control and an independent risk factor for diabetes complications [67]. Additionally, GV can be influenced by several nutritional factors, including carbohydrate quality, quantity, and distribution, as well as protein and fiber intake. These factors have important implications for clinical nutrition practice and diabetes management. Due to financial constraints, this kind of measurement could not have been applied in the present study. However, it would be appealing to obtain such data in future research. Nonetheless, the AID group participants achieved a greater decrease in $\mathrm{HbA} 1 \mathrm{c}$ level than that reported in the meta-analyses of the Mediterranean diet as a glycemic control diet [68-70]. Additionally, concerning comparable studies [20,37,65,71], reductions in the other glycemic parameters were similar.

The AID group participants significantly increased the proportion of total energy intake from fat, reaching $44 \%$. If it exceeds $35-40 \%$, it may affect insulin sensitivity [72]. However, total fats are the sum of different types of fatty acids, and the type of fatty acid is more important than their total amount [72]. The AID group significantly increased the intake of mono- and polyunsaturated fatty acids, particularly omega-3 fatty acids, and did not significantly decrease the level of glycated hemoglobin, which is similar to the results of the PREDIMED (Prevención con Dieta Mediterránea) sub-study [73], where dietary fat intake, including monounsaturated and omega-3 fatty acids, was associated with a higher risk of hyperglycemia. Dyslipidemia has been identified as a risk factor for cardiovascular disease and has been associated with hyperinsulinemia [74]. High concentrations of LDL-C and triglycerides are associated with a risk of cardiovascular disease, while HDL-C levels are the second substitute marker for other cause-and-risk factors of cardiovascular disease [75]. For these reasons, the focus on lowering only one of these biomarkers via dietary changes may affect the intake of certain food components and individuals may be deprived of its pleiotropic effects of the avoided foods, such as dairy food, which contains saturated fatty acids. The AID group participants significantly increased their HDL-C while the CD group participants achieved a significant reduction in their total cholesterol and LDL-C. This could be the effect of a significant reduction in total dietary fat intake and changes in the dietary fatty acid type. While participants in both treatment groups significantly reduced the proportion of total energy intake from saturated fatty acids, the AID group participants significantly increased their proportion of mono- and polyunsaturated fatty acids. Greater reductions in serum triglycerides, total cholesterol, and LDL-C were observed in both treatment arms of the present study than those reported in meta-analyses on the evidence of the efficacy of the Mediterranean diet for the primary prevention and control of cardiovascular diseases [46,71], and somewhat smaller compared to the results obtained by other similar studies [20,37,65,71]. However, the AID group achieved a greater increase in HDL-C concentration than that observed in similar studies $[20,37]$ and the aforementioned meta-analyses $[46,71]$. 
Wakabayashi and Daimon created the CMI by placing the ratio of triglycerides to HDL-C in a product with a waist-to-height ratio [36]. This index has been shown to be a good predictor of coronary artery disease and central components of metabolic syndrome [36]. Since most components of the CMI are the components of metabolic syndrome, a change in CMI is associated with changes in metabolic syndrome components. The AID group achieved a greater decrease in CMI than the $\mathrm{CD}$ group. This effect was particularly pronounced in the subgroup of participants with metabolic syndrome, who achieved the largest decrease in triglycerides, but also an increase in HDL-C and a greater decrease in total and visceral fat mass compared to the subgroup of participants without metabolic syndrome (data not shown). In the CD group, the largest decrease in CMI was achieved in the subgroup with class I obesity, who achieved the largest decrease in total and visceral fat mass, total cholesterol, and LDL-C. The results presented herein support the fact that the moderate weight loss in individuals with obesity was associated with a reduction in visceral adipose tissue, which in turn led to significant improvements in metabolic syndrome parameters and a reduced risk of coronary heart disease and type 2 diabetes [76] since, in our study, the number of participants fulfilling the criteria for metabolic syndrome decreased by one-third in both groups following the 6-month dietary intervention. Weight loss in individuals with obesity caused by an energy-restricted Mediterranean diet did not significantly increase serum vitamin $\mathrm{D}$ levels, as it was obtained with an energy-restricted ketogenic diet, where for every kilogram of body weight lost, serum vitamin D levels tripled after one year of adherence to diet [77]. Participants who followed a ketogenic diet achieved a significantly greater weight loss than those who followed a Mediterranean diet, which has been explained by a lower adherence to the Mediterranean diet [77]. However, the AID group in our study significantly increased their serum vitamin $\mathrm{D}$, which could be explained by an adequate intake of vitamin $\mathrm{D}$ from food and supplements. These findings require further investigation regarding the effect of vitamin D supplementation on weight loss.

Diet could be a relatively mild anti-inflammatory intervention, and if investigated in conditions of low-grade inflammation, it may cause mild changes that are difficult to detect [78], especially if weight loss is achieved at the same time. In studies evaluating the association between diet and inflammation, the most disruptive factor is weight loss [79], as inflammation status improvement in response to diet is shown to be dependent on weight loss $[79,80]$. Because weight loss could affect adipose tissue function and the inflammatory response, a recent review of the effects of whole foods and dietary patterns on circulating inflammatory markers focused on weight-stable adults with overweight and obesity [78]. Although foods and dietary patterns discussed in this review were not found to have significant effects on inflammatory markers in weight-stable individuals, it was difficult to distinguish beneficial or neutral effects because of the methodological inconsistencies between the included studies. Headlend et al. [81] showed that a weight loss of 5\% enables a reduction in biomarkers of cardiovascular disease risk, including inflammatory biomarker CRP. A 10\% reduction in body weight can decrease serum inflammatory markers by more than twofold and reduce risk factors for cardiovascular disease [81]. In the present study, the biomarkers of inflammation were significantly reduced in both groups, similarly or slightly greater in comparison to some other studies that evaluated the impact of diet on markers of inflammation [78,79]. However, the CD group participants reduced their hs-CRP and IL- 6 concentrations slightly more than the AID group participants, while the AID group participants achieved a greater reduction in TNF- $\alpha$. Both study groups achieved an equal reduction in dietary energy intake, causing a significant loss of total body weight and fat mass. The AID group reduced their total body weight slightly more than the CD group, while the reduction in total and visceral fat mass was slightly more pronounced in the CD group. It has been observed that the reduction of adipose tissue, particularly of visceral adipose tissue, in individuals with obesity, leads to decreased cytokine production [79]. In the present study, the reduction in biomarkers of inflammation could be more of a cause of reducing the total and visceral fat mass than the effect of food ingredients with anti-inflammatory properties. This is in line with review studies of the effects of weight loss and/or diet on the reduction of inflammation $[78,79]$, which concluded that a 
reduction in total body weight and fat mass following an energy-restricted diet has a significant effect on the reduction in inflammatory markers, while a diet with anti-inflammatory ingredients has only a slight effect on reducing inflammatory markers in weight-stable individuals. Regression analyses of our data showed that changing the diet into a diet with more anti-inflammatory potential was significantly associated only with lower values of IL-6 among the AID group. Other parameters showed a downward association without reaching statistical significance, which could be explained with better-controlled cardiometabolic parameters among the AID group participants than among the CD group. A significant association of lower DII ${ }^{\circledR}$ with lower values of insulin, HOMA-IR, triglycerides, and CMI index was observed among the CD group participants, which was similar to that reported by an Australian study evaluating the effect of a Mediterranean diet on patients with coronary heart disease [62].

\subsection{Strengths and Limitations}

Dietary interventions for obesity management in randomized clinical trials are generally less effective compared to pharmacological interventions or surgery. It is difficult to highlight the effective nutrient(s) of an applied diet due to its complex and still not yet fully investigated metabolism, as well as methodological difficulties regarding standardizing a diet and fully monitoring what participants consume [82]. Choosing an inappropriate period to reveal a clinical effect could be a study limitation [78]. To overcome these limitations, the length of the present study was set to be 6 months, which is consistent with similar dietary intervention trials $[20,37,65,71,77]$. Significantly more women participated in our study, which also could represent a study limitation regarding data interpretation, as study results could more refer to the female gender rather than males. Participant recruitment was done in an obesity outpatient clinic where more women applied more and were also more willing to be involved in the trial compared to men. Low levels of participant compliance in nutrition trials decrease the power to detect effects on specified endpoints, result in false-negative findings, and ultimately mean that the study is unable to provide evidence to support a potentially beneficial effect of an intervention. Therefore, to maximize and assess compliance with dietary interventions, detailed dietary instructions, frequent contacts with a dietitian, and controlling food diaries according to the specified recommendations using two diet-monitoring methods were provided. This study was also strengthened by detailed assessments of dietary intake that were clarified by the dietitian and comprehensive outcome measures, including the DII ${ }^{\circledR}$, which was not only confirmed as an effective tool for assessing the diet quality, but also for monitoring the change in diet toward a more anti-inflammatory diet.

\section{Conclusions}

An energy-restricted anti-inflammatory diet was shown to be effective in the management of obesity in younger adults. Significant reductions in body weight, BMI, total and visceral adipose tissue, and improvements in body composition, cardiometabolic, and inflammatory parameters were achieved, not only in the intervention anti-inflammatory diet group but also in the control group; this was done by applying the standard protocol for weight reduction based on the Mediterranean diet, which has been acknowledged as an anti-inflammatory diet per se. There are still few data that have been obtained from randomized clinical trials on the effects of dietary interventions on body composition and the metabolic complications of obesity; therefore, the results presented herein could advance current scientific knowledge in the field of inflammation, nutrition, and obesity management.

Supplementary Materials: The following are available online at http://www.mdpi.com/2072-6643/12/11/3583/s1, Table S1: The changes in the consumption of food items, Table S2: The distribution of participants according to BMI classification and metabolic syndrome at the end of the trial $(n=81)(n(\%))$, Table S3: The proportion of total energy intake from dietary components. 
Author Contributions: Conceptualization, G.K.J., and S.K.M.; methodology, G.K.J., I.M.-S., S.P.Ž., B.Š., and S.K.M.; formal analysis, G.K.J., S.P.Ž., and I.M.-S.; investigation, G.K.J., S.P.Ž., B.Š., and S.K.M.; data curation, D.R.; writing — original draft preparation, G.K.J.; writing—review and editing, G.K.J., S.K.M., and D.R.; visualization, G.K.J.; supervision, S.K.M.; funding acquisition, S.K.M. All authors have read and agreed to the published version of the manuscript.

Funding: This research was funded by the University of Rijeka, Croatia, grant number Uniri-biomed-18-269/1441.

Acknowledgments: The authors would like to acknowledge the Croatian Society for Diabetes and Metabolic Diseases of the Croatian Medical Association, the Clinical Hospital Rijeka, Croatia, for the approval of conducting the original trial, all the participants, as well as the staff at Clinical Hospital Rijeka, Croatia, who made this study possible to conduct.

Conflicts of Interest: Sanja Klobučar Majanović is the vice president of the Croatian Society for Diabetes and Metabolic Disorders of the Croatian Medical Association and the vice president of the Croatian Society for Obesity of the Croatian Medical Association. She serves as an Executive Committee member of the Croatian Endocrine Society. She has served as principal investigator or co-investigator in clinical trials of Eli Lilly, Merck in North America (MSD), and Sanofi Aventis. She has received honoraria for speaking or advisory board engagements and consulting fees from Abbott, AstraZeneca, Boehringer Ingelheim, Eli Lilly, Lifescan-Johnson \& Johnson, Novartis, Novo Nordisk, MSD, Merck Sharp \& Dohme, Mylan, Pliva, and Sanofi Aventis. Dario Rahelić is the director of the Vuk Vrhovac University Clinic for Diabetes, Endocrinology, and Metabolic Diseases at Merkur University Hospital, Zagreb, Croatia. He is the president of the Croatian Society for Diabetes and Metabolic Disorders of the Croatian Medical Association. He serves as an Executive Committee member of the Croatian Endocrine Society, Croatian Society for Obesity, and Croatian Society for Endocrine Oncology. He was a board member and secretary of IDF Europe, and currently, he is the chair of the IDF Young Leaders in Diabetes (YLD) Program. He has served as an Executive Committee member of the Diabetes and Nutrition Study Group of the European Association for the Study of Diabetes (EASD), and currently, he serves as an Executive Committee member of the Diabetes and Cardiovascular Disease Study Group of EASD. He has served as a principal investigator or co-investigator in clinical trials for AstraZeneca, Eli Lilly, MSD, Novo Nordisk, Sanofi Aventis, Solvay, and Trophos. He has received honoraria for speaking or advisory board engagements and consulting fees from Abbott, Amgen, AstraZeneca, Bauerfeund, Bayer, Boehringer Ingelheim, Eli Lilly, Lifescan-Johnson \& Johnson, Novartis, Novo Nordisk, MSD, Merck Sharp \& Dohme, Mylan, Pfizer, Pliva, Roche, Salvus, Sanofi Aventis, and Takeda. The other authors declare no conflict of interest. The funders had no role in the design of the study; in the collection, analyses, or interpretation of data; in the writing of the manuscript, or in the decision to publish the results.

\section{References}

1. Frühbeck, G.; Toplak, H.; Woodward, E.; Yumuk, V.; Maislos, M.; Oppert, J.M. Executive Committee of the European Association for the Study of Obesity. Obesity: The gateway to ill health-An EASO position statement on a rising public health, clinical and scientific challenge in Europe. Obes. Facts 2013, 6, 117-120. [CrossRef] [PubMed]

2. Yumuk, V.; Tsigos, C.; Fried, M.; Schindler, K.; Busetto, L.; Micic, D.; Toplak, H. Obesity Management Task Force of the European Association for the Study of Obesity. European Guidelines for Obesity Management in Adults. Obes. Facts 2015, 8, 402-424. [CrossRef] [PubMed]

3. Marques, A.; Peralta, M.; Naia, A.; Loureiro, N.; de Matos, M.G. Prevalence of adult overweight and obesity in 20 European countries, 2014. Eur. J. Public Health 2018, 28, 295-300. [CrossRef] [PubMed]

4. Saltiel, A.R.; Olefsky, J.M. Inflammatory mechanisms linking obesity and metabolic disease. J. Clin. Investig. 2017, 127, 1-4. [CrossRef] [PubMed]

5. Hotamisligil, G.S. Inflammation, metaflammation and immunometabolic disorders. Nature 2017, 542, $177-185$. [CrossRef] [PubMed]

6. McLaughlin, T.; Ackerman, S.E.; Shen, L.; Engleman, E. Role of innate and adaptive immunity in obesity-associated metabolic disease. J. Clin. Investig. 2017, 127, 5-13. [CrossRef]

7. Wu, H.; Ballantyne, C.M. Metabolic Inflammation and Insulin Resistance in Obesity. Circ. Res. 2020, 126, 1549-1564. [CrossRef]

8. Forsythe, L.K.; Wallace, J.M.; Livingstone, M.B. Obesity and inflammation: The effects of weight loss. Nutr. Res. Rev. 2008, 21, 117-133. [CrossRef]

9. Chait, A.; den Hartigh, L.J. Adipose Tissue Distribution, Inflammation and Its Metabolic Consequences, Including Diabetes and Cardiovascular Disease. Front. Cardiovasc. Med. 2020, 7, 22. [CrossRef]

10. Calder, P.C.; Ahluwalia, N.; Brouns, F.; Buetler, T.; Clement, K.; Cunningham, K.; Esposito, K.; Jönsson, L.S.; Kolb, H.; Lansink, M.; et al. Dietary factors and low-grade inflammation in relation to overweight and obesity. Br. J. Nutr. 2011, 106, S5-S78. [CrossRef] 
11. Giugliano, D.; Ceriello, A.; Esposito, K. The effects of diet on inflammation: Emphasis on the metabolic syndrome. J. Am. Coll. Cardiol. 2006, 48, 677-685. [CrossRef] [PubMed]

12. Casas, R.; Estruch, R. Dietary Patterns, Foods, Nutrients and Chronic Inflammatory Disorders. Immunome Res. 2016, 12, 122. [CrossRef]

13. Alkhatib, A.; Tsang, C.; Tiss, A.; Bahorun, T.; Arefanian, H.; Barake, R.; Khadir, A.; Tuomilehto, J. Functional Foods and Lifestyle Approaches for Diabetes Prevention and Management. Nutrients 2017, 9, 1310. [CrossRef] [PubMed]

14. Razquin, C.; Martinez-Gonzalez, M.A. A Traditional Mediterranean Diet Effectively Reduces Inflammation and Improves Cardiovascular Health. Nutrients 2019, 11, 1842. [CrossRef]

15. Netea, M.G.; Balkwill, F.; Chonchol, M.; Cominelli, F.; Donath, M.Y.; Giamarellos-Bourboulis, E.J.; Golenbock, D.; Gresnigt, M.S.; Heneka, M.T.; Hoffman, H.M.; et al. A guiding map for inflammation. Nat. Immunol. 2017, 18, 826-831. [CrossRef]

16. Mancini, J.G.; Filion, K.B.; Atallah, R.; Eisenberg, M.J. Systematic Review of the Mediterranean Diet for Long-Term Weight Loss. Am. J. Med. 2016, 129, 407-415. [CrossRef]

17. Martínez-González, M.A.; Gea, A.; Ruiz-Canela, M. The Mediterranean Diet and Cardiovascular Health. Circ. Res. 2019, 124, 779-798. [CrossRef]

18. Schwingshackl, L.; Hoffmann, G. Mediterranean dietary pattern, inflammation and endothelial function: A systematic review and meta-analysis of intervention trials. Nutr. Metab. Cardiovasc. Dis. 2014, 24, 929-939. [CrossRef]

19. Medina-Remón, A.; Casas, R.; Tressserra-Rimbau, A.; Ros, E.; Martínez-González, M.A.; Fitó, M.; Corella, D.; Salas-Salvadó, J.; Lamuela-Raventos, R.M.; Estruch, R.; et al. Polyphenol intake from a Mediterranean diet decreases inflammatory biomarkers related to atherosclerosis: A substudy of the PREDIMED trial. Br. J. Clin. Pharmacol. 2017, 83, 114-128.

20. Casas, R.; Sacanella, E.; Urpí-Sardà, M.; Corella, D.; Castaner, O.; Lamuela-Raventos, R.M.; Salas-Salvadó, J.; Martínez-González, M.A.; Ros, E.; Estruch, R. Long-Term Immunomodulatory Effects of a Mediterranean Diet in Adults at High Risk of Cardiovascular Disease in the PREvención con DIetaMEDiterránea (PREDIMED) Randomized Controlled Trial. J. Nutr. 2016, 146, 1684-1693. [CrossRef]

21. Dinu, M.; Pagliai, G.; Angelino, D.; Rosi, A.; Dall'Asta, M.; Bresciani, L.; Ferraris, C.; Guglielmetti, M.; Godos, J.; Del Bo, C.; et al. Effects of Popular Diets on Anthropometric and Cardiometabolic Parameters: An Umbrella Review of Meta-Analyses of Randomized Controlled Trials. Adv. Nutr. 2020, 11, 815-833. [CrossRef] [PubMed]

22. Jovanović, G.K.; Mrakovcic-Sutic, I.; Udović, I.Š.; Majanović, S.K. Evaluating the Effect of an Energy-Restricted Anti-Inflammatory Diet on Weight Loss, Body Composition, Cardiometabolic Risk Factors and Immune System Response in Younger Adults with Obesity: Study Protocol for a Randomized Controlled Trial. Eur. J. Integr. Med. 2020, 37, 101165. [CrossRef]

23. Alberti, K.G.; Eckel, R.H.; Grundy, S.M.; Zimmet, P.Z.; Cleeman, J.I.; Donato, K.A.; Fruchart, J.C.; James, W.P.; Loria, C.M.; Smith, S.C., Jr. Harmonizing the metabolic syndrome: A joint interim statement of the International Diabetes Federation Task Force on Epidemiology and Prevention; National Heart, Lung, and Blood Institute; American Heart Association; World Heart Federation; International Atherosclerosis Society; and International Association for the Study of Obesity. Circulation 2009, 120, 1640-1645. [PubMed]

24. Ministarstvo Zdravlja. Odluka o Standardu Prehrane Bolesnika u Bolnicama. Narodne Novine, 59 (on Croatian). 2015. Available online: https://narodne-novine.nn.hr/clanci/sluzbeni/2007_11_121_3528.html (accessed on 15 February 2019).

25. Mifflin, M.D.; St Jeor, S.T.; Hill, L.A.; Scott, B.J.; Daugherty, S.A.; Koh, Y.O. A new predictive equation for resting energy expenditure in healthy individuals. Am. J. Clin. Nutr. 1990, 51, 241-247. [CrossRef] [PubMed]

26. Shivappa, N.; Steck, S.E.; Hurley, T.G.; Hussey, J.R.; Hébert, J.R. Designing and developing a literature-derived, population-based dietary inflammatory index. Public Health Nutr. 2014, 17, 1689-1696. [CrossRef]

27. Kenđel Jovanović, G.; Pavičić Žeželj, S.; Klobučar Majanović, S.; Mrakovcic-Sutic, I.; Šutić, I. Metabolic syndrome and its association with the Dietary Inflammatory Index (DII)®in a Croatian working population. J. Hum. Nutr. Diet. 2020, 33, 128-137. [CrossRef]

28. Kaić-Rak, A.; Antonić, K. Tablice o Sastavu Namirnica I Pića; Zavod za zaštitu zdravlja Hrvatske: Zagreb, Croatia, 1990. (On Croatian). 
29. Frida Food Data; Version 1; National Food Institute, Technical University of Denmark: Kongens Lyngby, Denmark, 2015; Available online: http://frida.fooddata.dk (accessed on 11 March 2019).

30. U.S. Department of Agriculture, Agricultural Research Service, USDA Nutrient Data Laboratory. USDA National Nutrient Database for Standard Reference. Available online: https://ndb.nal.usda.gov/ndb/ (accessed on 11 March 2019).

31. Rothwell, J.A.; Perez-Jimenez, J.; Neveu, V.; Medina-Remon, A.; M’Hiri, N.; García-Lobato, P.; Manach, C.; Knox, C.; Eisner, R.; Wishart, D.S.; et al. Phenol-Explorer 3.0: A major update of the Phenol-Explorer database to incorporate data on the effects of food processing on polyphenol content. Database 2013, 2013, bat070. [CrossRef]

32. Haytowitz, D.B.; Wu, X.; Bhagwat, S. USDA Database for the Flavonoid Content of Selected Foods, Release 3.3. U.S. Department of Agriculture, Agricultural Research Service. Nutrient Data Lab. 2018. Available online: http://www.ars.usda.gov/nutrientdata/flav (accessed on 18 March 2019).

33. Gunathile, K.D.P.P.; Ranaweera, K.K.D.S.; Rupasinghe, H.P.V. Influence of boiling, steaming and frying of selected leafy vegetables on the in vitro anti-inflammation associated biological activities. Plants 2018, 7, 22. [CrossRef]

34. Rothwell, J.A.; Medina-Remón, A.; Pérez-Jiménez, J.; Neveu, V.; Knaze, V.; Slimani, N.; Scalbert, A. Effects of food processing on polyphenol contents: A systematic analysis using phenol-explorer data. Nutrition 2019, 58, 140-148. [CrossRef]

35. Matthews, D.R.; Hosker, J.P.; Rudenski, A.S.; Naylor, B.A.; Treacher, D.F.; Turner, R.C. Homeostasis model assessment: Insulin resistance and beta-cell function from fasting plasma glucose and insulin concentrations in man. Diabetologia 1985, 28, 412-419. [CrossRef] [PubMed]

36. Wakabayashi, I.; Daimon, T. The "cardiometabolic index" as a new marker determined by adiposity and blood lipids for discrimination of diabetes mellitus. Clin. Chim. Acta 2015, 438, 274-478. [CrossRef] [PubMed]

37. Salama, A.A.; Amine, E.K.; Salem, H.A.; Abd El Fattah, N.K. Anti-Inflammatory Dietary Combo in Overweight and Obese Women with Polycystic Ovary Syndrome. N. Am. J. Med. Sci. 2015, 7, 310-316. [CrossRef] [PubMed]

38. Cavallari, J.F.; Denou, E.; Foley, K.P.; Khan, W.I.; Schertzer, J.D. Different Th17 immunity in gut, liver, and adipose tissues during obesity: The role of diet, genetics, and microbes. Gut Microbes 2016, 7, 82-89. [CrossRef] [PubMed]

39. Gotsis, E.; Anagnostis, P.; Mariolis, A.; Vlachou, A.; Katsiki, N.; Karagiannis, A. Health benefts of the Mediterranean Diet: An Update of Research Over the Last 5 Years. Angiology 2015, 66, 304-318. [CrossRef] [PubMed]

40. Romagnolo, D.F.; Selmin, O.I. Mediterranean Diet and Prevention of Chronic Diseases. Nutr. Today 2017, 52, 208-222. [CrossRef] [PubMed]

41. Salas-Salvado, J.; Becerra-Tomas, N.; García-Gavilán, J.F.; Bullo, M.; Barrubes, L. Mediterranean Diet and Cardiovascular Disease Prevention: What Do We Know? Prog. Cardiovasc. Dis. 2018, 61, 62-67. [CrossRef] [PubMed]

42. Andreoli, A.; Lauro, S.; Di Daniele, N.; Sorge, R.; Celi, M.; Volpe, S.L. Effect of a moderately hypoenergetic Mediterranean diet and exercise program on body cell mass and cardiovascular risk factors in obese women. Eur. J. Clin. Nutr. 2008, 62, 892-897. [CrossRef]

43. Franquesa, M.; Pujol-Busquets, G.; García-Fernández, E.; Rico, L.; Shamirian-Pulido, L.; Aguilar-Martínez, A.; Medina, F.X.; Serra-Majem, L.; Bach-Faig, A. Mediterranean Diet and Cardiodiabesity: A Systematic Review through Evidence-Based Answers to Key Clinical Questions. Nutrients 2019, 11, 655. [CrossRef]

44. Ortner Hadžiabdić, M.; Vitali Čepo, D.; Rahelić, D.; Božikov, V. The Effect of the Mediterranean Diet on Serum Total Antioxidant Capacity in Obese Patients: A Randomized Controlled Trial. J. Am. Coll. Nutr. 2015, 35, 224-351. [CrossRef]

45. Estruch, R. Anti-inflammatory effects of the Mediterranean diet: The experience of the PREDIMED study. Proc. Nutr. Soc. 2010, 69, 333-340. [CrossRef] [PubMed]

46. Rees, K.; Takeda, A.; Martin, N.; Ellis, L.; Wijesekara, D.; Vepa, A.; Das, A.; Hartley, L.; Stranges, S. Mediterranean-style diet for the primary and secondary prevention of cardiovascular disease. Cochrane Database Syst. Rev. 2019, 3, CD009825. [CrossRef] [PubMed]

47. Doko Jelinić, J.; Pucarin-Cvetković, J.; Nola, I.A.; Senta, A.; Milošević, M.; Kern, J. Regional differences in dietary habits of adult Croatian population. Coll. Antropol. 2009, 33, 31-34. 
48. Kenđel Jovanović, G.; Pavičić Žeželj, S.; Malatestinić, Đ.; Mrakovčić Šutić, I.; Nadarević Štefanac, V.; Dorčić, F. Diet quality of middle age and older women from Primorsko-Goranska County evaluated by healthy eating index and association with body mass index. Coll. Antropol. 2010, 34, 155-160.

49. Pavičić Žeželj, S.; Kenđel Jovanović, G.; Dragaš Zubalj, N.; Mićović, V.; Sesar, Ž. Associations between Adherence to the Mediterranean Diet and Lifestyle Assessed with the MEDLIFE Index among the Working Population. Int. J. Environ. Res. Public Health 2018, 15, 2126. [CrossRef]

50. Sahay, R.D.; Couch, S.C.; Missoni, S.; Sujoldžić, A.; Novokmet, N.; Duraković, Z.; Rao, M.B.; Musić Milanović, S.; Vuletić, S.; Deka, R.; et al. Dietary patterns in adults from an Adriatic Island of Croatia and their associations with metabolic syndrome and its components. Coll. Antropol. 2013, 37, 335-342.

51. Tomas, Ž.; Škarić-Jurić, T.; Zajc Petranović, M.; Jalšovec, M.; Rajić Šikanjić, P.; Smolej Narančić, N. Waist to height ratio is the anthropometric index that most appropriately mirrors the lifestyle and psychological risk factors of obesity. Nutr. Diet. 2019, 76, 539-545. [CrossRef]

52. Willoughby, D.; Hewlings, S.; Kalman, D. Body Composition Changes in Weight Loss: Strategies and Supplementation for Maintaining Lean Body Mass, a Brief Review. Nutrients 2018, 10, 1876. [CrossRef]

53. Connaughton, R.M.; McMorrow, A.M.; McGillicuddy, F.C.; Lithander, F.E.; Roche, H.M. Impact of anti-inflammatory nutrients on obesity-associated metabolic-inflammation from childhood through to adulthood. Proc. Nutr. Soc. 2016, 75, 115-124. [CrossRef]

54. Perez-Jimenez, J.; Fezeu, L.; Touvier, M.; Arnault, N.; Manach, C.; Hercberg, S.; Galan, P.; Scalbert, A. Dietary intake of 337 polyphenols in French adults 1-3. Am. J. Clin. Nutr. 2011, 93, 1220-1228. [CrossRef]

55. Hostetler, G.L.; Ralston, R.A.; Schwartz, S.J. Flavones: Food Sources, Bioavailability, Metabolism, and Bioactivity. Adv. Nutr. 2017, 8, 423-435. [CrossRef] [PubMed]

56. The EFSA Comprehensive European Food Consumption Database. Available online: http://data.europa.eu/ euodp/data/dataset/the-efsa-comprehensive-european-food-consumption-database (accessed on 15 June 2019).

57. WHO: GEMS/Food Consumption Database. Available online: https://www.who.int/nutrition/landscape analysis/nlis_gem_food/en/ (accessed on 17 June 2019).

58. Tapsell, L.C.; Hemphill, I.; Cobiac, L.; Sullivan, D.R.; Fenech, M.; Patch, C.S.; Roodenrys, S.; Keogh, J.B.; Clifton, P.M.; Williams, P.G.; et al. Health benefits of herbs and spices: The past, the present, the future. Med. J. Aust. 2006, 1854, S4-S24. [CrossRef]

59. Jiang, T.A. Health Benefits of Culinary Herbs and Spices. J. AOAC Int. 2019, 102, 395-411. [CrossRef] [PubMed]

60. Shoba, G.; Joy, D.; Joseph, T.; Rajendran, M.M.; Srinivas, P.S. Influence of piperine on the pharmacokinetics of curcumin in animals and human volunteers. Planta Med. 1998, 64, 353-356. [CrossRef] [PubMed]

61. Babatunde, O.A.; Arp Adams, S.; Truman, S.; Sercy, E.; Murphy, A.E.; Khan, S.; Hurley, T.G.; Wirth, M.D.; Choi, S.K.; Johnson, H.; et al. The impact of a randomized dietary and physical activity intervention on chronic inflammation among obese African-American women. Women Health 2020, 1-14. [CrossRef]

62. Mayr, H.L.; Itsiopoulos, C.; Tierney, A.C.; Ruiz-Canela, M.; Hebert, J.R.; Shivappa, N.; Thomas, C.J. Improvement in dietary inflammatory index score after 6-month dietary intervention is associated with reduction in interleukin-6 in patients with coronary heart disease: The AUSMED heart trial. Nutr. Res. 2018, 55, 108-121. [CrossRef]

63. Turner-McGrievy, G.M.; Wirth, M.D.; Shivappa, N.; Dunn, C.G.; Crimarco, A.; Hurley, T.G.; West, D.S.; Hussey, J.R.; Hébert, J.R. Impact of a 12-month Inflammation Management Intervention on the Dietary Inflammatory Index, inflammation, and lipids. Clin. Nutr. ESPEN 2019, 30, 42-51. [CrossRef]

64. Wirth, M.D.; Shivappa, N.; Khan, S.; Vyas, S.; Beresford, L.; Sofge, J.; Hébert, J.R. Impact of a 3-Month Anti-inflammatory Dietary Intervention Focusing on Watermelon on Body Habitus, Inflammation, and Metabolic Markers: A Pilot Study. Nutr. Metab. Insights 2020, 13, 1178638819899398. [CrossRef]

65. Jovanović, Ž.; Crnčević-Orlić, Ž.; Štimac, D.; Kokić, S.; Peršić, V.; Ružić, T.; Goll-Barić, S. Effects of obesity reduction on cardiovascular risk factors: Comparison of individual and group treatment-substudy of the Croatian Healthy Weight Loss Programme. Coll. Antropol. 2009, 33, 751-757.

66. Hoyas, I.; Leon-Sanz, M. Nutritional Challenges in Metabolic Syndrome. J. Clin. Med. 2019, 8, 1301. [CrossRef]

67. Tay, J.; Thompson, C.H.; Brinkworth, G.D. Glycemic Variability: Assessing Glycemia Differently and the Implications for Dietary Management of Diabetes. Ann. Rev. Nutr. 2015, 35, 389-424. [CrossRef] [PubMed]

68. Boucher, J.L. Mediterranean Eating Pattern. Diabetes Spectr. 2017, 30, 72-76. [CrossRef] [PubMed] 
69. Hallberg, S.J.; Dockter, N.E.; Kushner, J.A.; Athinarayanan, S.J. Improving the scientific rigour of nutritional recommendations for adults with type 2 diabetes: A comprehensive review of the American Diabetes Association guideline-recommended eating patterns. Diabetes Obes. Metab. 2019, 21, 1769-1779. [CrossRef] [PubMed]

70. Wheeler, M.L.; Dunbar, S.A.; Jaacks, L.M.; Karmally, W.; Mayer-Davis, E.J.; Wylie-Rosett, J.; Yancy, W.S. Macronutrients, food groups, and eating patterns in the management of diabetes: A systematic review of the literature, 2010. Diabetes Care 2012, 35, 434-445. [CrossRef] [PubMed]

71. Huo, R.; Du, T.; Xu, Y.; Xu, W.; Chen, X.; Sun, K.; Yu, X. Effects of Mediterranean-style diet on glycemic control, weight loss and cardiovascular risk factors among type 2 diabetes individuals: A meta-analysis. Eur. J. Clin. Nutr. 2015, 69, 1200-1208. [CrossRef] [PubMed]

72. Vessby, B.; Uusitupa, M.; Hermansen, K.; Riccardi, G.; Rivellese, A.A.; Tapsell, L.C.; Nälsén, C.; Berglund, L.; Louheranta, A.; Rasmussen, B.M.; et al. Substituting dietary saturated for monounsaturated fat impairs insulin sensitivity in healthy men and women: The KANWU Study. Diabetologia 2001, 44, 312-319. [CrossRef] [PubMed]

73. Julibert, A.; Bibiloni, M.D.; Bouzas, C.; Martínez-González, M.Á.; Salas-Salvadó, J.; Corella, D.; Zomeño, M.D.; Romaguera, D.; Vioque, J.; Alonso-Gómez, Á.M.; et al. Total and Subtypes of Dietary Fat Intake and Its Association with Components of the Metabolic Syndrome in a Mediterranean Population at High Cardiovascular Risk. Nutrients 2019, 11, 1493. [CrossRef]

74. Savva, S.C.; Lamnisos, D.; Kafatos, A.G. Predicting cardiometabolic risk: Waist-to-height ratio or BMI. A meta-analysis. Diabetes Metab. Syndr. Obes. 2013, 6, 403-419. [CrossRef]

75. Wu, J.H.Y.; Micha, R.; Mozaffarian, D. Dietary fats and cardiometabolic disease: Mechanisms and effects on risk factors and outcomes. Nat. Rev. Cardiol. 2019, 16, 581-601. [CrossRef]

76. Després, J.P. Is visceral obesity the cause of the metabolic syndrome? Ann. Med. 2006, 38, 52-63. [CrossRef]

77. Perticone, M.; Maio, R.; Sciacqua, A.; Suraci, E.; Pinto, A.; Pujia, R.; Zito, R.; Gigliotti, S.; Sesti, G.; Perticone, F. Ketogenic Diet-Induced Weight Loss is Associated with an Increase in Vitamin D Levels in Obese Adults. Molecules 2019, 24, 2499. [CrossRef] [PubMed]

78. Cowan, S.F.; Leeming, E.R.; Sinclair, A.; Dordevic, A.L.; Truby, H.; Gibson, S.J. Effect of whole foods and dietary patterns on markers of subclinical inflammation in weight-stable overweight and obese adults: A systematic review. Nutr. Rev. 2020, 78, 19-38. [CrossRef] [PubMed]

79. Bianchi, V.E. Weight loss is a critical factor to reduce inflammation. Clin. Nutr. ESPEN 2018, 28, 21-35. [CrossRef] [PubMed]

80. Magkos, F.; Fraterrigo, G.; Yoshino, J.; Luecking, C.; Kirbach, K.; Kelly, S.C.; De Las Fuentes, L.; He, S.; Okunade, A.L.; Patterson, B.W.; et al. Effects of Moderate and Subsequent Progressive Weight Loss on Metabolic Function and Adipose Tissue Biology in Humans with Obesity. Cell Metab. 2016, 23, 591-601. [CrossRef] [PubMed]

81. Headland, M.; Clifton, P.M.; Carter, S.; Keogh, J.B. Weight-Loss Outcomes: A Systematic Review and Meta-Analysis of Intermittent Energy Restriction Trials Lasting a Minimum of 6 Months. Nutrients 2016, 8, 354. [CrossRef]

82. Woodside, J.V.; Welch, R.W.; Patterson, C.C.; McKinley, M.C. Study Design: Intervention Studies. In Nutrition Research Methodologies; Lovegrove, J.A., Hodson, L., Sharma, S., Lanham-New, S.A., Eds.; The Nutrition Society Textbook Series; Wiley-Blackwell: West Sussex, UK, 2015; pp. $28-47$.

Publisher's Note: MDPI stays neutral with regard to jurisdictional claims in published maps and institutional affiliations.

(C) 2020 by the authors. Licensee MDPI, Basel, Switzerland. This article is an open access article distributed under the terms and conditions of the Creative Commons Attribution (CC BY) license (http://creativecommons.org/licenses/by/4.0/). 